\title{
An Energy-Based Nonlinear Dynamic Model of Dielectric Elastomer Minimum Energy Structures with Stiffeners: Equilibrium Configuration and the Electromechanical Response
}

\author{
Aman Khurana \\ Indian Institute of Technology Roorkee \\ Asesh Patra \\ Indian Institute of Technology Roorkee \\ M M Joglekar ( $\nabla$ manish.joglekar@me.iitr.ac.in ) \\ Indian Institute of Technology Roorkee https://orcid.org/0000-0003-0525-3923
}

\section{Research Article}

Keywords: Dielectric elastomer minimum energy structure (DEMES), Stiffeners, Equilibrium configuration, Nonlinear dynamics, Phase portraits

Posted Date: October 21st, 2021

DOl: https://doi.org/10.21203/rs.3.rs-991095/v1

License: (c) (1) This work is licensed under a Creative Commons Attribution 4.0 International License.

Read Full License 


\title{
An Energy-based Nonlinear Dynamic Model of Dielectric Elastomer Minimum Energy Structures with Stiffeners: Equilibrium Configuration and the Electromechanical Response
}

\author{
Aman Khurana · Asesh Patra · M. M. \\ Joglekar*
}

Received: date / Accepted: date

\begin{abstract}
The Dielectric Elastomer-based Minimum Energy Structures (DEMES) pertain to an equilibrium configuration attained by the assembly of a pre-stretched electroactive polymer film and a compliant boundary frame. Because of their unique characteristics, such as fast response and a large reversible stroke; DEMES have been used widely in the development of soft robotic transducers. However, their utility is typically restricted because of the warping resulting from anticlastic curvature. The present investigation examines the effectiveness of stiffeners in controlling this warping, as well as their effect on the resulting electromechanical response when the DEMES is driven electrically. To this end, we devise an energy-based analytical model that predicts the initial equilibrium configuration of an elementary rectangular DEMES with a finite number of stiffeners adhered to the boundary frame. The proposed framework uses the neo-Hookean hyperelasticity model for the polymer film and the linear elastic constitutive model for both the frame and the stiffeners. Predictive capability of the proposed analytical model is established through comparisons with 3D finite element simulations and experimental observations. The analytical model is then extended in the setting of the least-action principle to investigate the complex nonlinear dynamic behavior of the DEMES emanating from the interplay between material and geometric nonlinearities. The proposed dynamic model provides crucial insights into the role of varying levels of geometric and material parameters on the attainment of the initial equilibrium configuration of DEMES and its DC dynamic response when driven by a Heaviside electric load. In particular, we highlight the favorable impact of adding stiffeners in enhancing the stroke of the DEMES and an amplification in the attained equilibrium angle with an increasing spacing between the stiffeners. The analytical model and the results reported in this
\end{abstract}

Aman Khurana, Asesh Patra, M. M. Joglekar*

Department of Mechanical and Industrial Engineering, Indian Institute of Technology Roorkee

Roorkee, Uttarakhand, India. 247667

E-mail: akhurana1@me.iitr.ac.in, akumarpatra@me.iitr.ac.in, manish.joglekar@me.iitr.ac.in 
investigation can be of potential use in pre-designing the geometrical and material properties of DEMES for enhancing its electromechanical performance.

Keywords Dielectric elastomer minimum energy structure (DEMES) - Stiffeners . Equilibrium configuration $\cdot$ Nonlinear dynamics $\cdot$ Phase portraits

\section{Introduction}

Due to their characteristics of undergoing large reversible areal deformations (more than $100 \%)$ Pelrine et al. (2000), the Dielectric elastomers (DEs) have been incorporated constructively in minimum energy structures Kofod et al. (2006). DE based minimum energy structures resemble a 3-D equilibrium configuration delineated by the minimum energy state of the system Kofod et al. (2007). The DEMES is formed by the combination of an inextensible frame and a pre-stretched DE membrane. As the structure is released from external mechanical constraint, the restoring force of the membrane bends the elastic frame into a minimum energy configuration. Further, due to the applied electric voltage across the electrodes of the membrane, the frame flattens out. The electrically induced bending of the compliant frame, acquirement of the primary configuration after removal of the voltage, and the electric voltage-driven bi-stability are certain essential deformation modes of the DEMES actuator which is exploited constructively to develop grippers Kofod et al. (2007), flapping-wing actuators Liu et al. (2013); Zhao et al. (2015a), compliant mechanisms Gu et al. (2017); Ma et al. (2013); Zhao et al. (2015b), etc.

In most of the aforementioned DEMES applications, the dynamic motion strongly affects their performance. Hence, modeling the nonlinear dynamic responses of the DEMES actuator is an important task for the effective design of such devices. Specifically, for the dynamic behavior of the planar DEAs, several investigations considering the effect of prestressing or pre-stretch Dai and Wang (2015); Joglekar (2014); Sharma et al. (2017), viscoelasticity Kollosche et al. (2015); Wang et al. (2014); Zhao et al. (2020), humidityZhang et al. (2021), the initial stretch ratio Dai et al. (2016), combined DC and AC voltage Khurana et al. (2021a); Sasikala et al. (2017); Sheng et al. (2014) on the electromechanical behavior of the DEAs are available. Realistically, the inherent material nonlinearity of the DE membrane is scrutinized in all the above-stated work of DEAs. However, the DE-based minimum energy structures exhibit very complex and rich behavior due to the interplay of geometric and constitutive nonlinearities. Among available few research related to the dynamic behavior of DEMES actuators, O'brien et al. O'Brien et al. (2009), developed a finite element model to interrogate the quasi-static behaviors of the DEMES. Zhao et al. Zhao et al. (2015b) presented an experimental investigation depicting the behavior of a DEMES rotary joint actuated by a square form voltage signal. Further, Zhao et al. Zhao et al. (2016b) presented a semi-analytical model based on the linear equation of motion to study the behavior of rotary joint DEMES. Recently, few researchers presented a lumped parameter modeling of DE-based minimum energy structures to provide insights on the nonlinear dynamic behavior of the DEMES actuators Khurana et al. (2020, 2021b); Vatanjou et al. (2019). 
Apart from the dynamic behavior, the DEMES needs enough deformation range in the desired direction to achieve the vibrational spectrum of the actuation devices, specifically for soft robotic applications. However, the desired in-plane deformation of the DEMES is typically restricted by warping (out-of-plane deformation) of the structure. Several techniques in the recent past have been developed to circumvent such problems, e.g., providing pure shear pre-stretching condition to the membrane Rosset et al. (2014), incorporating anisotropy to the material behavior of the DE membrane to promote bending of the DEMES in required direction Khurana et al. (2021b), Implementing a stiffening frame along with the compliant frame to provide rigidity and avoid the unnecessary out-of-plane bending of the DEMES Zhao et al. (2016a), etc. In context of DEMES, providing initial pure shear prestretching conditions instead of equal bi-axial stretching to the membrane promotes the bending of the DEMES predominantly along the desired length direction and the parasitic bending along the width direction can be controlled easily by strengthening the frame extremities portion of the rectangular shaped bending actuator Rosset et al. (2014). However, achieving the pure shear prestretching of the DE membrane involves a tedious fabrication process Araromi et al. (2014). For planar DE actuator, Lai et. al Lai et al. (2012) presented a multi-layer dielectric elastomer actuator design, reinforced with periodic stiffeners to achieve the complex desired deformation of the DE actuator. To the best of our knowledge the effect of anisotropy, imparted by finitely spaced reinforcements on controlling the warping, and subsequently actuation performance of DEMES has not been investigated so far.

This work first provides an experimentally and numerically validated static analytical framework for assessing the equilibrium configuration of the proposed DEMES. The developed static analytical framework effectively predicts the attained equilibrium angle of the DEMES. Next, we extend the proposed analytical framework to investigate the complex nonlinear dynamic behavior of the DEMES. In the dynamic analysis, a detailed study for extracting the influence of various material and geometrical parameters of the DEMES is presented. Further, we analyse the effect of spacing between the consecutive stiffeners implemented on the surface of compliant frame on the attained initial and final configuration of the DEMES actuator. The proposed investigation implicates that imparting reinforcements on the surface of inextensible frame significantly controls the warping and actuation behavior of the DEMES actuator. The analytical framework developed herein can assist the designing of practical devices for energy harvesting and actuation purposes.

The remaining paper is structured as follows: Section 2, discusses the outlook of the proposed dielectric elastomer based minimum energy structures. Static analysis pertains to the development of analytical, experimental and numerical model to investigate the attained equilibrium state of the proposed DEMES is reported in Section 3. The analytical framework based on the deduced nonlinear governing equation of motion to investigate the complex nonlinear dynamic behavior of the DEMES actuator is presented in Section 4. A comprehensive parametric investigation revealing the impact of frame geometrical parameters and incorporated stiffeners on the performance of DEMES is presented in Section 5. Finally, Section 6 summarizes the salient findings of the present study. 


\section{Problem Definition}

This article presents the incorporation of 3-D reinforcements on the compliant frame to reduce the warping effect and obtain the desired initial as well as final configuration of the DEMES. Fig. 1 elucidates the notion of warping in DEMES actuators using the initial equilibrium configurations predicted numerically using 3-D FE simulations performed in ABAQUS. As evident from the Figure, the DEMES with reinforcements (Fig. 1 b) shows a considerable reduction in warping when compared with the one without reinforcements (Fig. 1 a). The considered DEMES consists of a compliant frame of initial length $L_{1}$, width $W_{1}$, and thickness $h$, respectively, with a rectangular hole as shown schematically in Fig. 2. The characterizing dimensions of the rectangular cut section of the compliant frame are length $C$, width $W$, and thickness $h$, respectively. An equally bi-axially pre-stretched membrane (Fig. 2) having the same dimensions (length $L_{1}$, and width $W_{1}$ ) as that of the compliant frame is affixed to the frame to develop the proposed minimum energy structure.

Further, the 3-D reinforcements are attached to the surface of the frame to avoid the warping of the structure, as depicted in Fig. 2. Finally, a stiffening frame towards the fixed end of the compliant frame is attached to provide rigidity towards the end portion of the frame. The associated dimensions characterizing the developed DEMES are shown schematically in Fig. 2 and corresponding values are indexed in

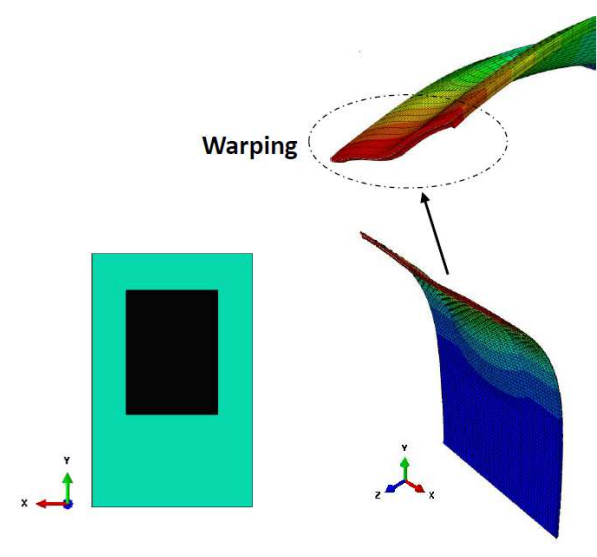

(a) DEMES: Without Reinforcement

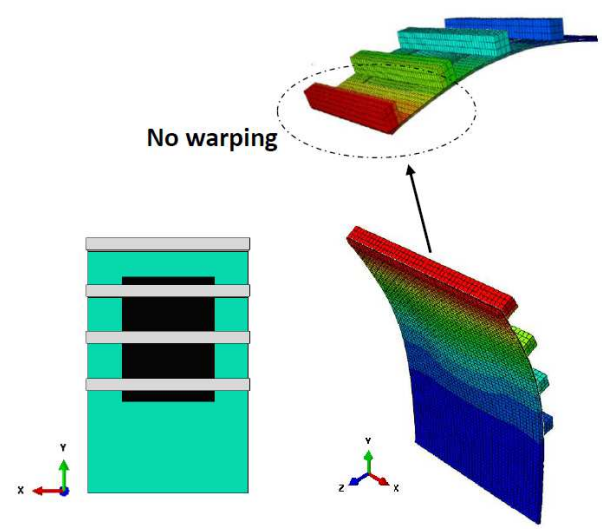

(b) DEMES: With Reinforcement

Fig. 1 Numerically (ABAQUS) obtained initial configuration of the DEMES (a) without reinforcement (showing warping), and (b) with reinforcement (no warping).

Table 1 Geometrical dimensions of the parts incorporated to form the DEMES.

\begin{tabular}{cccc}
\hline Part & Length $(\mathrm{cm})$ & Width $(\mathrm{cm})$ & Thickness $(\mathrm{mm})$ \\
\hline Compliant Frame & $L_{1}=5.50, C=2.70$ & $W_{1}=3.50, W=2.00$ & $h=0.40$ \\
Elastomer Membrane & $L_{1}=5.50$ & $W_{1}=3.50$ & $t_{1}=f\left(\lambda_{p}\right)$ \\
3D Stiffeners & $a_{1}=0.20$ & $W_{1}=3.50$ & $a_{2}=2.50$ \\
Stiffening Frame & $L_{0}=2.0$ & $W_{1}=3.50$ & $h=0.40$ \\
\hline
\end{tabular}




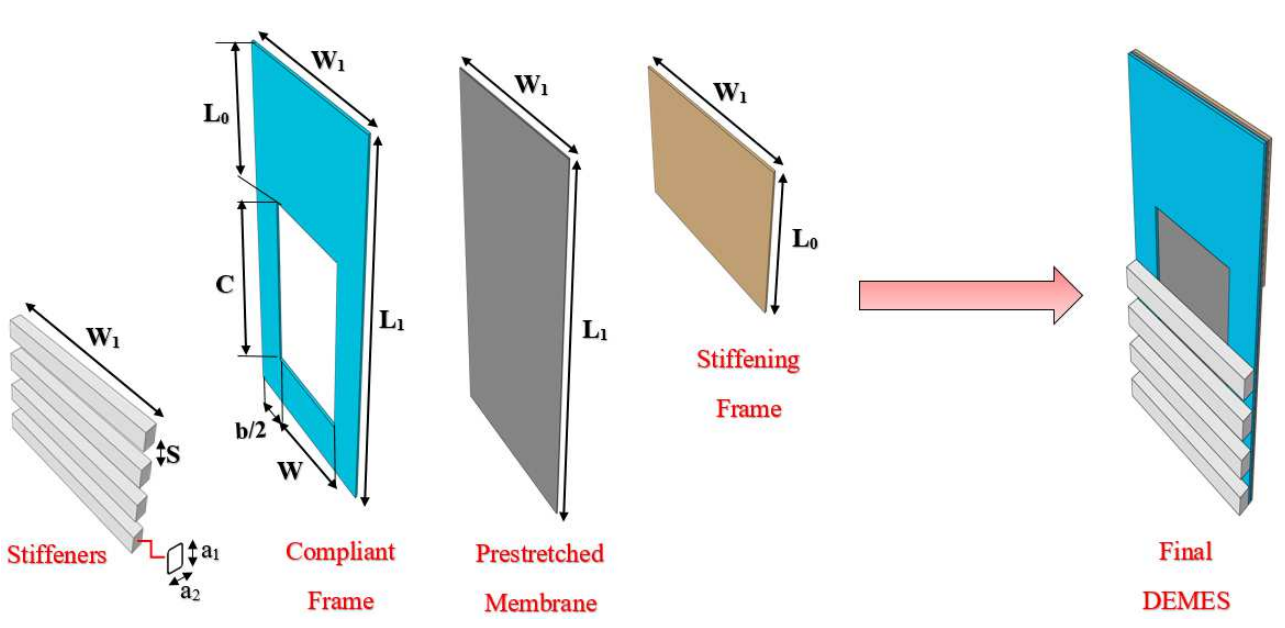

Fig. 2 Problem definition: Schematic of the proposed DEMES.

Table 1 for a ready reference. The bending strips width $(b)$ and the thickness $(h)$ of the compliant frame are the critical dimensions that can be tuned to alter the stiffness of the compliant frame. The spacing $(S)$ between the adjacent stiffeners also affects the initial and the final state of the developed DEMES actuator.

The upcoming section presents the static analysis of the proposed DEMES to investigate the attained equilibrium configuration of the structure.

\section{Static analysis of DE based minimum energy structures}

One of the crucial states of the DEMES is referred to as the initial configuration which is obtained as a consequence of energy redistribution between the elastomer film and the deformable frame when the DEMES disengages from external mechanical constraint. This initial or equilibrium configuration of the DEMES is characterized by equilibrium angle denoted as $\theta_{e q}$.

In the following subsections, we present the analytical, experimental and numerical methodology implemented for the measurement of equilibrium angle of the proposed DE based minimum energy structure.

\subsection{Analytical modeling of DEMES}

This subsection provides a static analytical model to analyse the equilibrium configuration of the proposed DEMES utilizing the principle of minimization of the total potential energy of the system.

The proposed analytical model of the DEMES is shown schematically in Fig. 3. To evaluate the associated strain energy of the stretched membrane, it is assumed that the membrane spans across the two extremities edge of the frame as illustrated in 
(a)

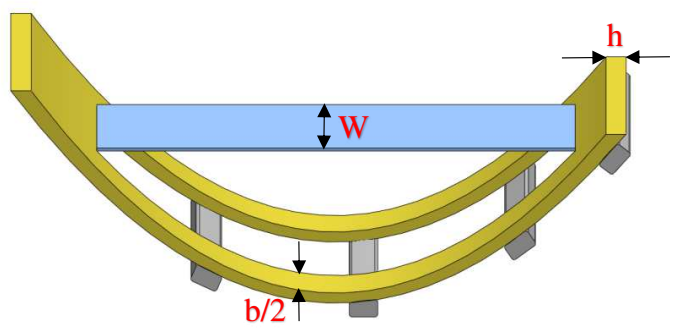

(c)

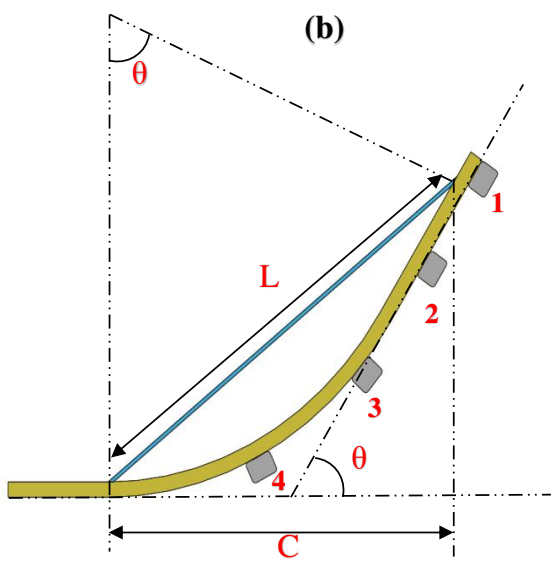

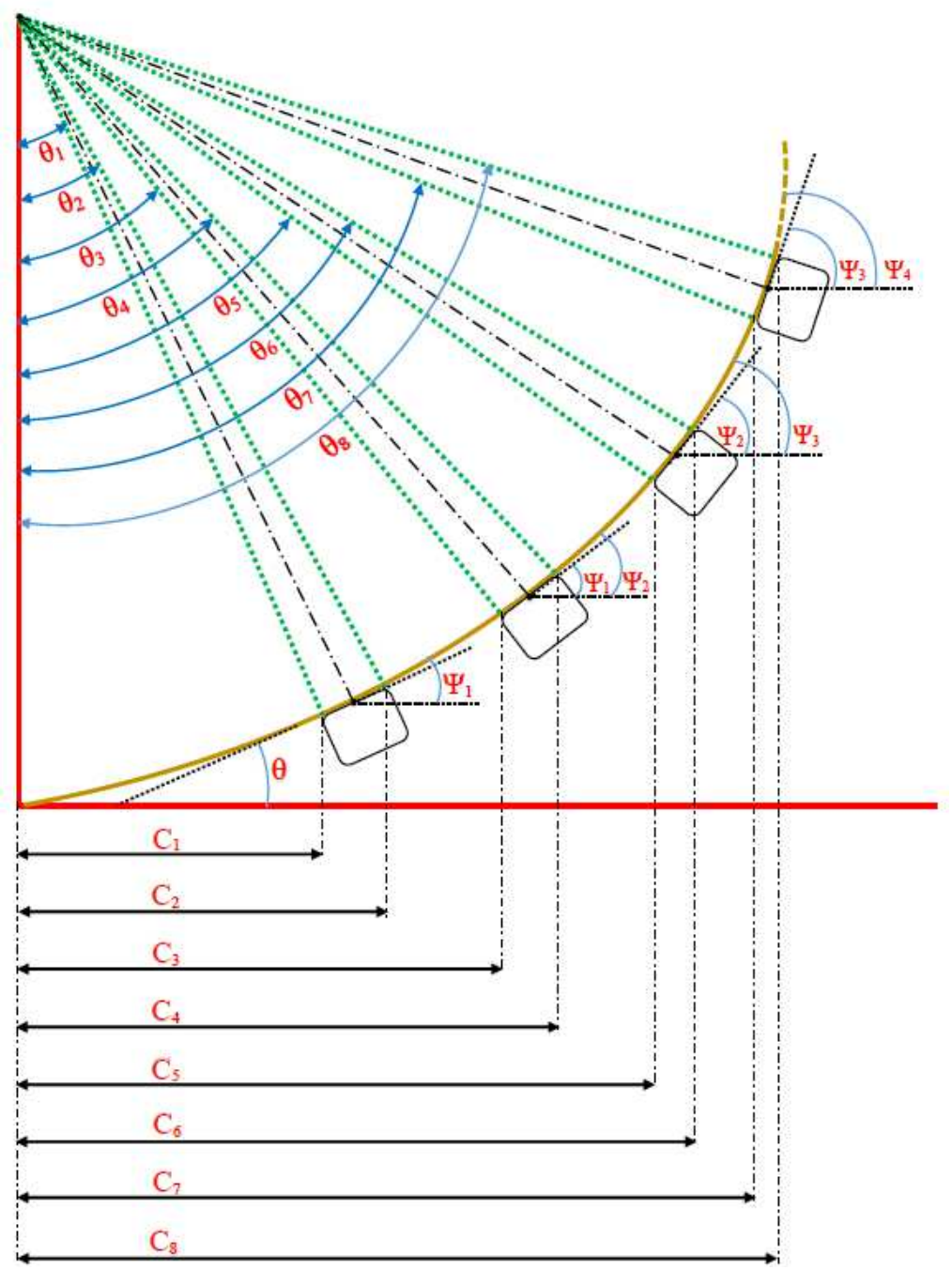

Fig. 3 Schematic of the DEMES representing the geometrical dimensions in (a) Front view, (b) Side view, and (c) Enlarged side view. 
Fig. 3 a,b. However, the membrane also deforms with the compliant frame in actual practice and forms a complex saddle shape. This hypothesis facilitates the lumped parameter modeling of the DE-based minimum energy structures and has been applied constructively in the recent past Khurana et al. (2021b); Rosset et al. (2014); Vatanjou et al. (2019). To evaluate the associated energies of the developed DEMES, we discretized the entire DEMES based on the implemented stiffeners as shown in Fig. 3 c. The expressions for the different curved lengths and angles corresponding to the embedded stiffeners are obtained geometrically from Fig. $3 \mathrm{c}$ and provided in the Appendix A for a ready reference.

\subsubsection{Bending energy of frame with embedded stiffeners}

In the developed DEMES, the frame is an important part characterized by two bending strips having width $b / 2$ each and the thickness $h$ as revealed in Fig. 3 a. The DE membrane enforces a constant bending moment to the compliant frame and thereby causes the frame to bend along length $C$ of the rectangular hole. Therefore, the energy required to bend the frame is expressed as

$$
U_{1}=\int_{0}^{C} \frac{M^{2} d x}{2 E_{f} I}=\frac{E_{f} b h^{3}}{24 C}(\theta)^{2}=\frac{K_{b f}}{2}(\theta)^{2},
$$

in which $K_{b f}$ represents the associated compliant frame bending stiffness which can be defined in terms of mechanical and geometrical parameters of the frame as $K_{b f}=\frac{E_{f} b h^{3}}{12 C}$, where $E_{f}$ represents the Young's modulus of the frame.

To avoid the warping of the DEMES, the stiffeners are also attached to the surface of the compliant frame (Fig. 3). However, on the downside, it enhances the bending stiffness of the frame by the expression:

$$
U_{2}=\frac{K_{b s}}{2}\left(\frac{W_{s}}{C^{2}}\right)\left[\left(2 C-W_{s}\right)+\sum_{n=2}^{N}\left(2 C-W_{s}-n S\right)\right](\theta)^{2}
$$

in which $K_{b s}$ is the bending stiffness of the stiffener, $W_{s}$ represents the width of the stiffener, $n$ is the number of stiffeners, $N$ represents the maximum number of stiffeners, and $S$ denotes the spacing between the consecutive stiffeners, respectively. In the present study, we keep the number of stiffeners $N=4$ for all the analysis. Hence, upon simplification, the Eq. (2) for $N=4$ stiffeners is rewritten as

$$
U_{2}=\underbrace{\frac{K_{b s}}{2}\left(\frac{W_{s}}{C^{2}}\right)\left(\begin{array}{l}
\left(2 C-W_{s}\right)+\left(2 C-W_{s}-2 S\right) \\
+\left(2 C-W_{s}-3 S\right)+\left(2 C-W_{s}-4 S\right)
\end{array}\right)}_{\text {Stiffeners }}(\theta)^{2}
$$

The total energy required to bend the compliant frame having stiffeners embedded on the surface is obtained by adding Eq. (1) and Eq. (3) as 
152

$$
\begin{aligned}
& U_{\text {total }}=U_{1}+U_{2}, \\
& U_{\text {total }}=\underbrace{\frac{K_{b f}}{2}(\theta)^{2}}_{\text {frame }}+\underbrace{\frac{K_{b s}}{2}\left(\frac{W_{s}}{C^{2}}\right)\left(\begin{array}{l}
\left(2 C-W_{s}\right)+\left(2 C-W_{s}-2 S\right) \\
+\left(2 C-W_{s}-3 S\right)+\left(2 C-W_{s}-4 S\right)
\end{array}\right)}_{\text {Stiffeners }}(\theta)^{2}
\end{aligned}
$$

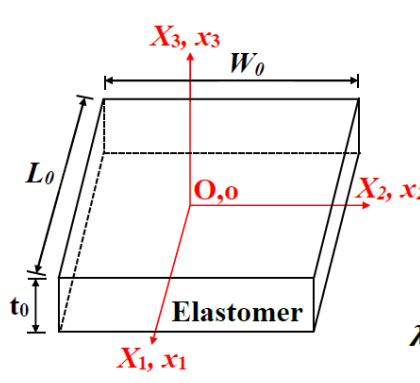

(a)

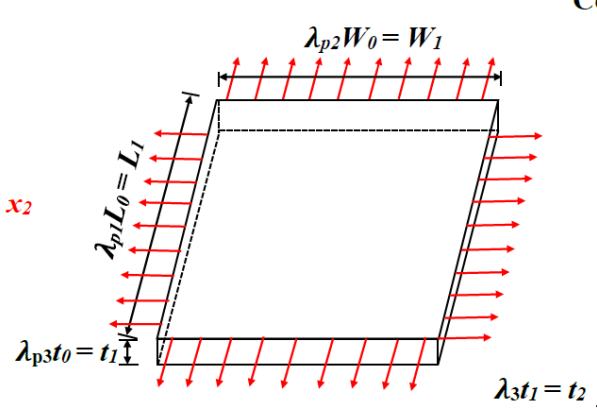

(b)
Compliant electrode

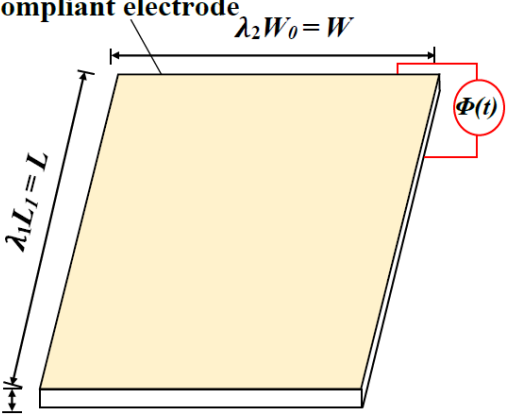

(c)

Fig. 4 Schematic of DE membrane in (a) Initial state, (b) Prestretched state, and (c) actuated state.

\subsubsection{Strain energy of membrane}

The schematic of the DE membrane attached to the inextensible frame to develop the required DEMES is shown in Fig. 4. In the initial configuration, the membrane has the initial dimensions of $L_{0} \times W_{0} \times t_{0}$ in the $X_{1}, X_{2}$, and $X_{3}$ directions (Fig. 4 a), respectively. Due to equal biaxial pre-stretching of the membrane in $X_{1}$ and $X_{2}$ directions, the dimensions of the membrane become $L_{1} \times W_{1} \times t_{1}$, respectively. By invoking the incompressibility condition of the DE membrane, the developed prestretches in the principal directions of the DE membrane are drafted as

$$
\lambda_{p 1}=\lambda_{p}=\frac{L_{1}}{L_{0}}=\frac{C}{L_{0}} ; \quad \lambda_{p 2}=\lambda_{p}=\frac{W_{1}}{W_{0}} ; \lambda_{p 3}=\frac{1}{\lambda_{p 1} \lambda_{p 2}}
$$

where $\lambda_{p 1}, \lambda_{p 2}$, and $\lambda_{p 3}$ denote the developed pre-stretch in $X_{1}, X_{2}$, and $X_{3}$ directions, respectively, $L_{1}$ represents the length of the pre-stretched membrane equal to the length $C$ of a rectangular hole in the frame. The obtained stretches in Eq. (5) correspond to the developed initial stretch state in which the compliant frame remains flat. Due to applied potential difference $\phi(t)$ between the two electrodes of the membrane, the expansion of the DE membrane along $X_{1}$ and $X_{2}$ directions, and concomitant reduction in thickness, i.e., along $X_{3}$ direction occurs. Due to the implication of the incompressible nature of the DE membrane $\left(\lambda_{1} \lambda_{2} \lambda_{3}=1\right)$, the developed true stretches in the principal directions of the membrane are expressed as 


$$
\lambda_{1}=\frac{L}{L_{0}} ; \quad \lambda_{2}=\frac{W}{W_{0}} ; \quad \lambda_{3}=\frac{1}{\lambda_{1} \lambda_{2}},
$$

in which $\lambda_{1}, \lambda_{2}$, and $\lambda_{3}$ denote the developed true pre-stretches in $X_{1}, X_{2}$, and $X_{3}$ directions, respectively.

The dimensions of the membrane in the actuated configuration becomes $L \times W \times$ $t_{2}$, respectively as shown in Fig. 4 c. From Fig. 3 b, the DE membrane length $(L)$ is expressed in terms of bending angle $\left(\theta_{X 1}=\theta\right)$ of the frame in the $X_{1}$ direction as

$$
L=\frac{2 L_{1}}{\theta_{X 1}} \sin \left(\frac{\theta_{X 1}}{2}\right)=\frac{2 C}{\theta} \sin \left(\frac{\theta}{2}\right) .
$$

Similarly, the DE membrane width $(W)$ is expressed in terms of bending angle $\left(\theta_{X 2}\right)$ of the frame in the $X_{2}$ direction as

$$
W=\frac{2 W_{1}}{\theta_{X 2}} \sin \left(\frac{\theta_{X 2}}{2}\right) .
$$

The bending of the frame along width direction is negligible, hence invoking the assumption of $\sin \left(\frac{\theta_{X 2}}{2}\right) \approx \frac{\theta_{X 2}}{2}$. The expression for width $(W)$ of the membrane (Eq. 8) becomes

$$
W=\frac{2 W_{1}}{\theta_{X 2}} \sin \left(\frac{\theta_{X 2}}{2}\right)=\frac{2 W_{1}}{\theta_{X 2}} \times\left(\frac{\theta_{X 2}}{2}\right)=W_{1}
$$

On inserting the expressions of length $L$ (Eq. (7)), and width $W$ (Eq. (9)) in Eq. (6), the developed true stretches in the membrane is expressed as a function of bending angle $(\theta)$ of the compliant frame as

$$
\lambda_{1}=\frac{L}{L_{0}}=\frac{2 C}{\theta L_{0}} \sin \left(\frac{\theta}{2}\right) ; \lambda_{2}=\frac{W}{W_{0}}=\frac{W_{1}}{W_{0}} ; \lambda_{3}=\frac{1}{\lambda_{1} \lambda_{2}} .
$$

Using the expressions of $\lambda_{p 1}$ and $\lambda_{p 2}$ from Eq. 5 in Eq. 10, the final expressions for the developed true stretch state in the DE membrane is articulated as the function of the prestretch $\left(\lambda_{p}\right)$ of the membrane and bending angle $(\theta)$ of the frame as

$$
\lambda_{1}=\frac{2 \lambda_{p}}{\theta} \sin \left(\frac{\theta}{2}\right) ; \lambda_{2}=\lambda_{p} ; \lambda_{3}=\frac{1}{\lambda_{1} \lambda_{2}} .
$$

In this investigation, the generalized neo-Hookean material model Colonnelli et al. (2015); Luo et al. (2020) is used to describe the hyperelastic response of the membrane. However, this choice is not restrictive and the analysis presented here can easily be extended for assessing the effect of other models of hyperelasticity, such as Mooney-Rivlin Joglekar (2014); Soares et al. (2020), YeohChen et al. (2018); Wissler and Mazza (2005), Gent Zhang and Chen (2020); Zhang et al. (2018); Zhou et al. (2018) and Arruda-Boyce Park et al. (2012), etc. Based on the neo-Hookean hyperelastic material model, the expression for the strain energy density of the DE membrane is written as 


$$
\psi_{\text {membrane }}=\frac{\mu}{2}\left(\lambda_{1}^{2}+\lambda_{2}^{2}+\lambda_{3}^{2}-3\right)
$$

in which $\mu$ is the shear modulus of the DE membrane. Upon inheriting the expressions of $\lambda_{1}, \lambda_{2}$, and $\lambda_{3}$ from Eq. 11 and substituted in Eq. 12, the expression for the strain energy density of the membrane is drafted as

$$
\psi_{\text {membrane }}=\frac{\mu}{2}\left(\frac{4 \lambda_{p}^{2}}{\theta^{2}} \sin ^{2}\left(\frac{\theta}{2}\right)+\lambda_{p}^{2}+\frac{\theta^{2}}{4 \lambda_{p}^{4} \sin ^{2}\left(\frac{\theta}{2}\right)}-3\right)
$$

In the present study, an ideal dielectric model is adopted Tang et al. (2017), i.e., the permittivity $(\varepsilon)$ of the DE membrane is considered constant and independent of mechanical deformation and electric displacement. The electrical free energy of the DE membrane using the ideal dielectric model is expressed as Rosset et al. (2014)

$$
\psi_{\text {electrical }}=-\frac{1}{2} \varepsilon E^{2} \lambda_{1}^{2} \lambda_{2}^{2}=-\frac{\varepsilon E^{2}}{2} \frac{4 \lambda_{p}^{4}}{\theta^{2}} \sin ^{2}\left(\frac{\theta}{2}\right),
$$

Here, $\varepsilon$ represents the membrane permittivity, $E$ signifies the developed nominal electric field in the membrane obtained as $E=\frac{\phi(t)}{t_{1}}$, in which $\phi(t)$ denotes the potential difference applied across the membrane, and $t_{1}$ represent the pre-stretched membrane thickness. The total free energy density expression of the DE membrane is articulated as

$$
\begin{aligned}
& \psi=\psi_{\text {membrane }}+\psi_{\text {electrical }} \\
& \psi=\left[\begin{array}{l}
\underbrace{\frac{\mu}{2}\left(\frac{4 \lambda_{p}^{2}}{\theta^{2}} \sin ^{2}\left(\frac{\theta}{2}\right)+\lambda_{p}^{2}+\frac{\theta^{2}}{4 \lambda_{p}^{4} \sin ^{2}\left(\frac{\theta}{2}\right)}-3\right)}_{\text {membrane }} \\
-\underbrace{\frac{\varepsilon E^{2}}{2} \frac{4 \lambda_{p}^{4}}{\theta^{2}} \sin ^{2}\left(\frac{\theta}{2}\right)}_{\text {electrical }}
\end{array}\right]
\end{aligned}
$$

The expression for the total free energy associated with the membrane in the actuated configuration is evaluated by taking the volume integral of the total strain energy density $(\psi)$ from Eq. (15) as

$$
U_{\text {membrane }}=\int_{V} \psi d V=W_{0} L_{0} t_{0}[\underbrace{\left.\frac{\mu\left(\frac{4 \lambda_{p}^{2}}{\theta^{2}} \sin ^{2}\left(\frac{\theta}{2}\right)+\lambda_{p}^{2}+\frac{\theta^{2}}{4 \lambda_{p}^{4} \sin ^{2}\left(\frac{\theta}{2}\right)}-3\right)}{-\underbrace{\frac{\varepsilon E^{2}}{2} \frac{4 \lambda_{p}^{4}}{\theta^{2}} \sin ^{2}\left(\frac{\theta}{2}\right)}_{\text {electrical }}}\right]}_{\text {membrane }}]
$$


The total potential energy of the considered DEMES is expressed as the summation of total bending energy of frame with embedded stiffeners (Eq. (4)) and the total free energy of the DE membrane (Eq. (16)) and is written as

$$
U=U_{\text {total }}+U_{\text {membrane }}
$$

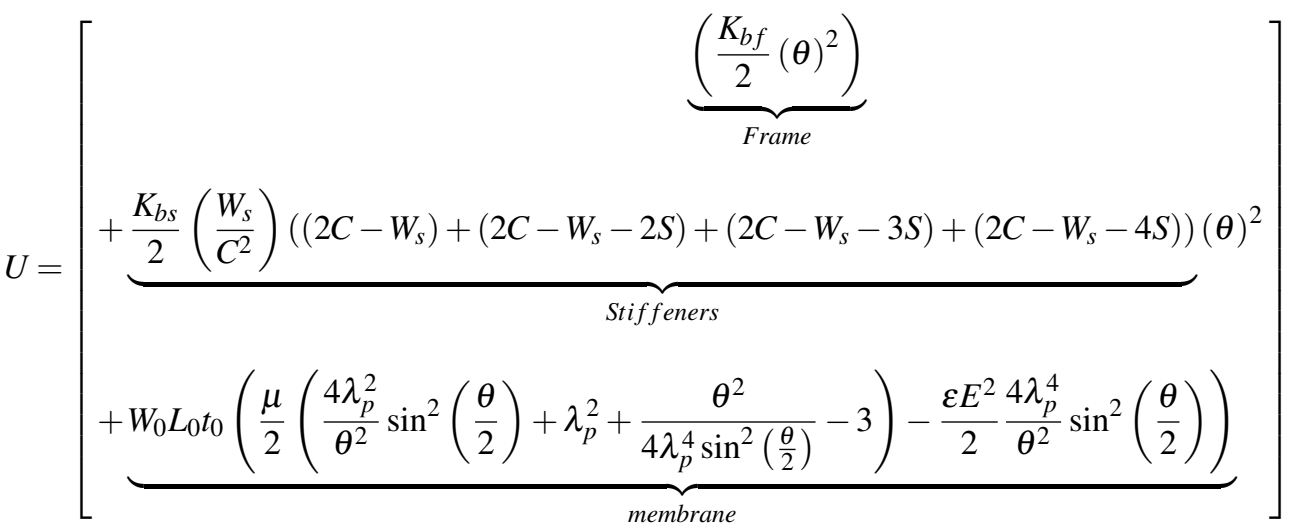

The static behavior of the DEMES is obtained by equating the first derivative of the total potential energy $(U)$ with bending angle $\theta$ equal to zero, i.e., $\frac{d U}{d \theta}=0$.

\subsection{Experimental analysis of DEMES}

In the experimental study, a commercially available acrylic elastomer (VHB-4910) with an initial thickness of $1 \mathrm{~mm}$, PET (polyethylene terephthalate) compliant frame of thickness $0.4 \mathrm{~mm}$, and 3D printed PLA (polyactic acid) reinforcements are used to form the required DE based minimum energy structures. The working mechanism implemented experimentally to develop the required DEMES is shown in Fig. 5. First of all, a DE membrane of initial dimensions $10 \mathrm{~cm} \times 10 \mathrm{~cm} \times 1 \mathrm{~mm}$, is attached to the mechanism as shown in Fig. 5 a. An equal biaxial prestretch is provided to the membrane by utilizing the stretching mechanism (Fig. 5 b). Next, a compliant PET frame having a rectangular hole at its center is attached to the stretched membrane. The rectangular-shaped compliant electrodes are produced at the center portion of the membrane using carbon grease (MG chemicals, 846-80G). Copper strips are used to make the connection of the electrodes with the input voltage terminals. The 3D PLA reinforcements are attached on the surface of the compliant frame using an adhesive (Arclear 92892). Finally, a stiffening frame made up of PET material is attached towards the end portion of the compliant frame to develop the proposed DEMES, as shown in Fig. 5 d. The mechanically constrained DEMES in undeformed configuration is shown in Fig. 5 e. An exploded view of the stretching mechanism used for providing equal bi-axial prestretch to the membrane is revealed in Fig. 6 a. To experimentally investigate the effect of spacing $(S)$ between the adjacent stiffeners on the performance of DEMES, we implemented four different DEMES with varying 


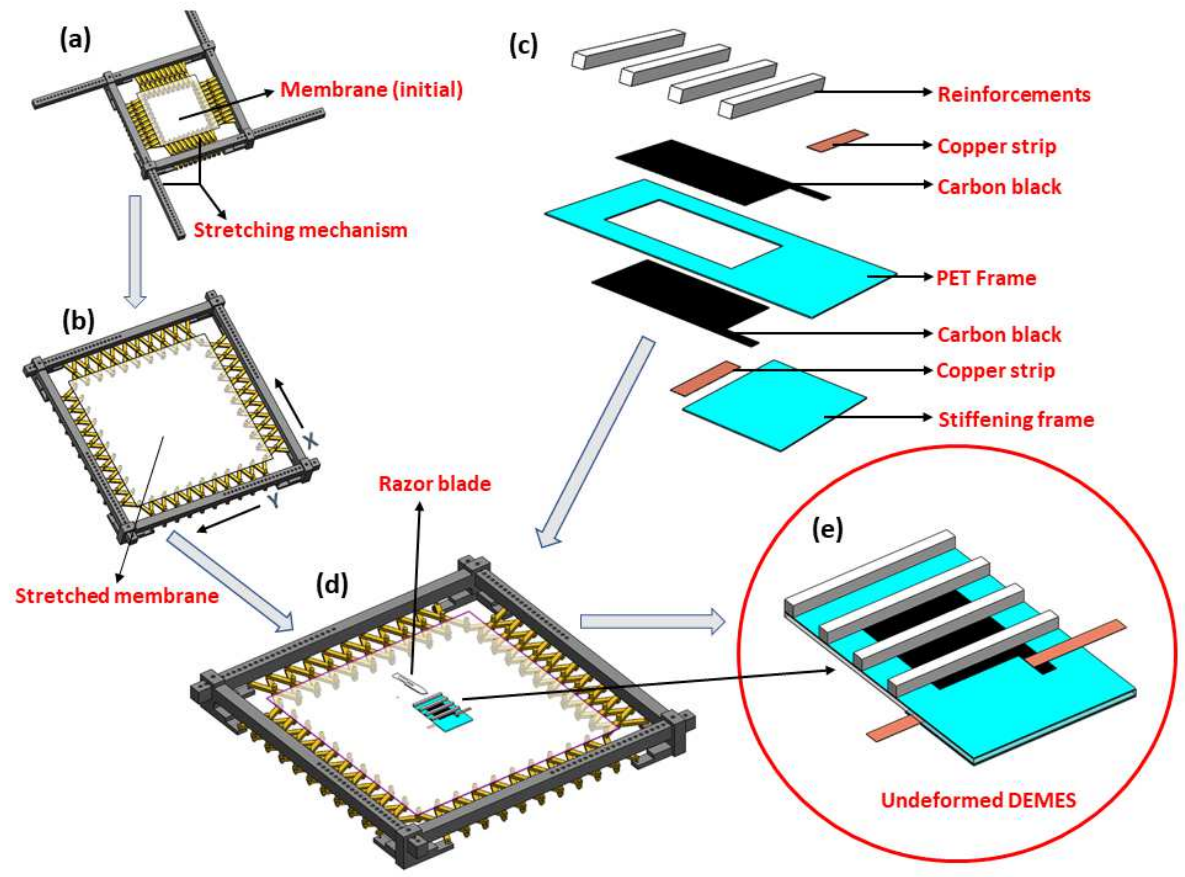

Fig. 5 Experimental working mechanism implemented to obtain the proposed DEMES.

spacing $(S)$ together, as shown in Fig. 6 a. A partition tape is implemented for easy removal of all the DEMES. Finally, the DEMES is removed from the constrained mechanism with the help of a razor blade. Due to redistribution of the energy among the structure, a newly deformed structure in the bend configuration is obtained. Figure $6 \mathrm{~b}$ presents the experimentally obtained bend configurations of the four different DEMES having varying levels of spacing $(S)$ between the adjacent stiffeners. From Fig. 6 b, it is evident that the bending of the structure increases with the increase in spacing $(S)$ between the stiffeners.

Figure 7 shows the methodology implemented for measuring the equilibrium angle of the DEMES. The steps implemented in the image processing technique utilizing MATLAB code are sequentially illustrated as follows: First, the experimentally obtained image of the DEMES with green color marks at the end of the stiffeners (Fig. 7 a) is considered as an input image of the DEMES in the developed image processing code. The input image is filter out to obtain the green channel image as depicted in Fig. 7 b. Further, the input image of the DEMES is converted to a grayscale image, as shown in Fig. 7 c. The obtained grayscale image is subtracted from the green channel image to get the end cross-section view of the stiffeners (Fig. $7 \mathrm{~d}$ ). In the next step, the small objects are filter out and the obtained image depicting the cross-section view of the stiffeners is converted to a binary image, as shown in Fig. 7 e. From the obtained binary image, the contours and their centroids are evaluated, and the obtained 


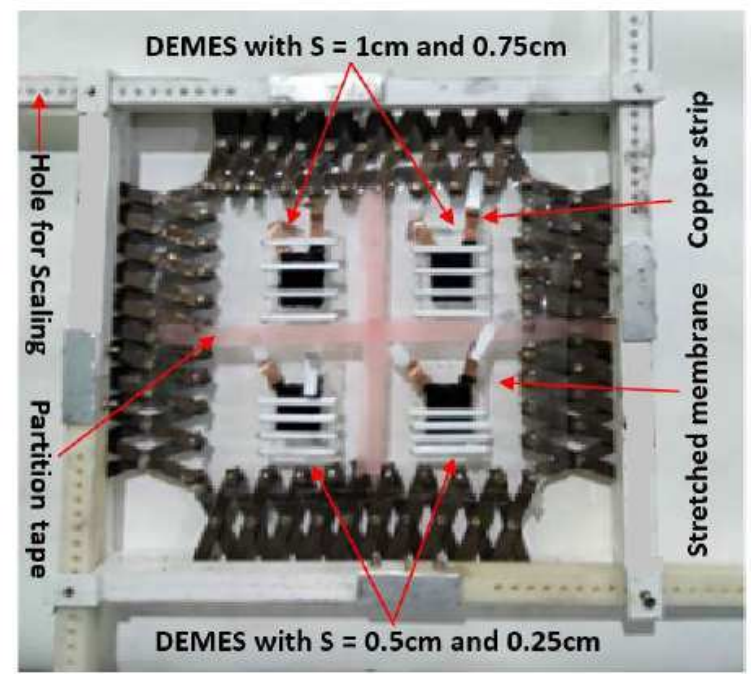

(a) Experimental: Stretching Mechanism

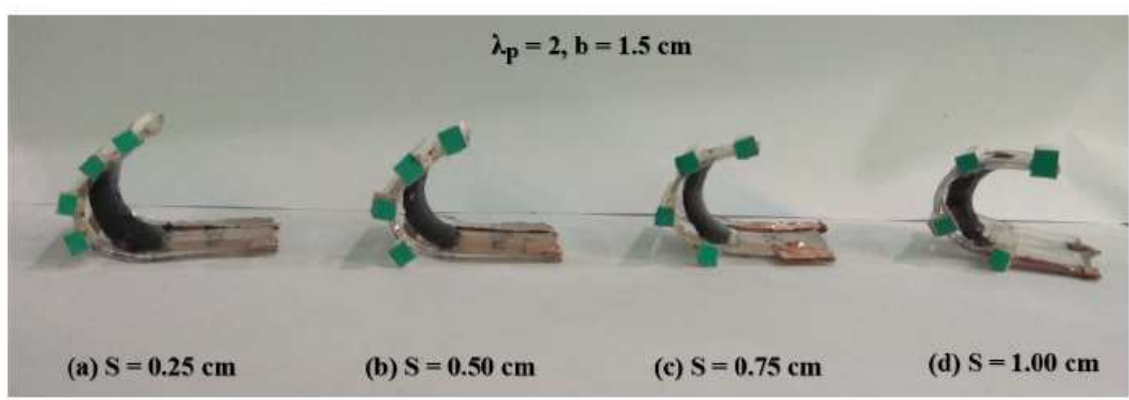

(b) Equilibrium configuration of the DEMES

Fig. 6 Experimental (a) Stretching mechanism, (b) Effect of spacing $(S)$ between the adjacent stiffeners on the attained equilibrium state of the DEMES.

centroids are represented with the help of points in the image (Fig. $7 \mathrm{f}$ ). The acquired centroid points are joined by using red color lines, and reference lines represented by green color intersects both the lines as depicted in Fig. 7 g. Finally, the attained equilibrium angle of the DEMES is evaluated as $\theta_{e q} .=180-\left(\theta_{1}-\theta_{2}\right)$, in which $\theta_{1}$, $\theta_{2}$ represents the obtained top and bottom angle in an anticlockwise direction to the reference lines in Fig. $7 \mathrm{~g}$. 


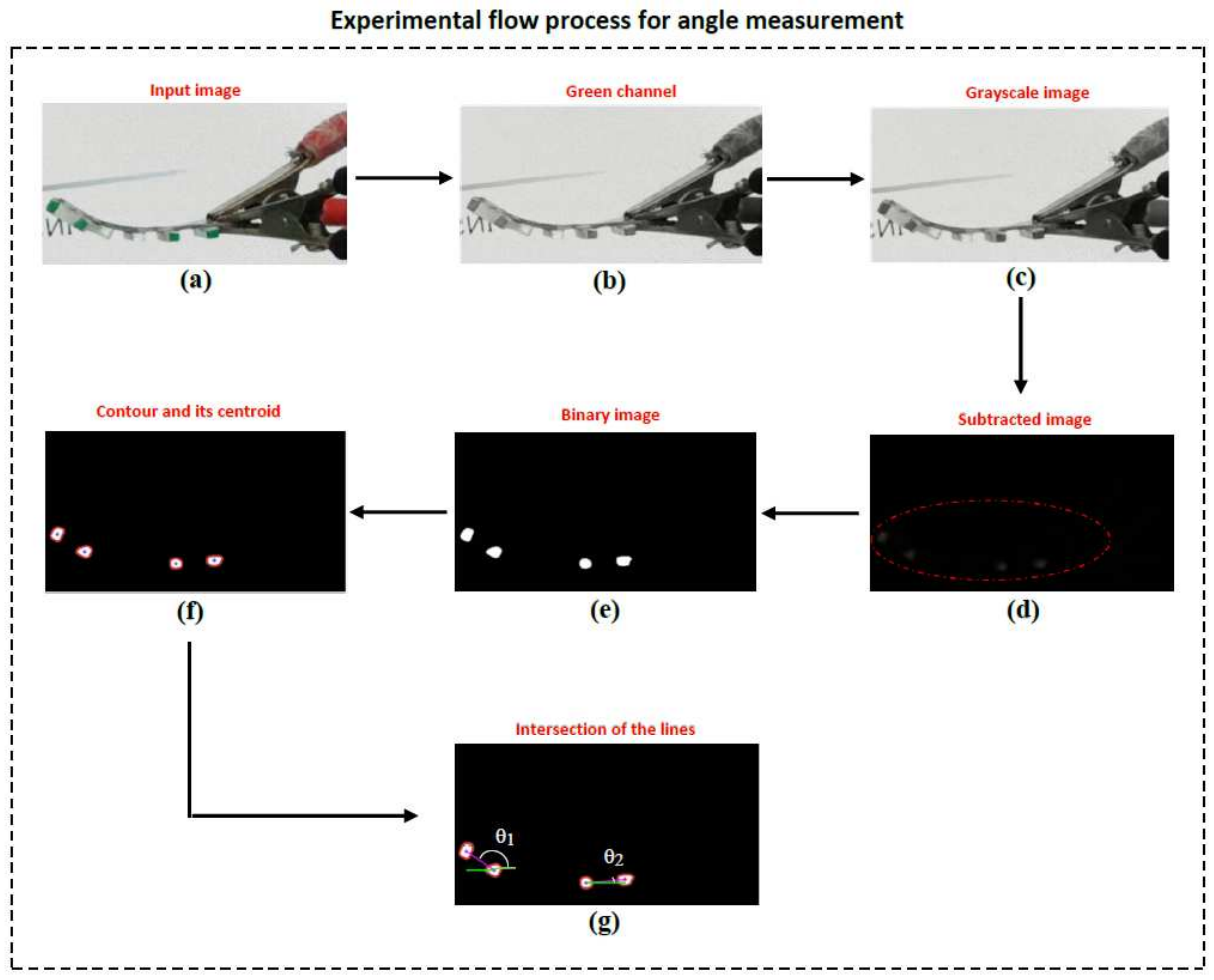

Fig. 7 Flow process representation to measure the attained equilibrium angle of the DEMES: (a) Input image of DEMES, (b) Filter image: Green channel, (c) Convert to a grayscale image, (d) Subtracting grayscale from green channel image, (e) Filter small objects and make image binary, (f) Finding centroid of contours and extracting points from contours, (g) Drawing of lines from points and intersect them with the reference lines.

\subsection{Finite element modeling of DEMES}

This subsection presents the finite element framework implemented using ABAQUS 6.19 to measure the equilibrium angle of the proposed DEMES.

\subsubsection{Kinematics}

Consider a continuum rectangular hyperelastic membrane $\mathscr{B}_{i}$ of thickness $t_{i}$ in the reference configuration with dimensions $L_{x i}$ and $L_{y i}$ in $x$ and $y$ directions, respectively. Also, $\frac{t_{i}}{L_{x i}}, \frac{t_{i}}{L_{y i}}<<1$ such that the deformed membrane can be delineate by the theory of thin hyperelastic membranes under finite deformations. Due to prestretching along $x$ and $y$ directions, the membrane attains the final configuration $\mathscr{B}_{f}$ of thickness $t_{f}$ with dimensions $L_{x f}$ and $L_{y f}$ in $x$ and $y$ directions as shown schematically in Fig. 8. A strain energy density function $\psi$ per unit initial volume of membrane can be 


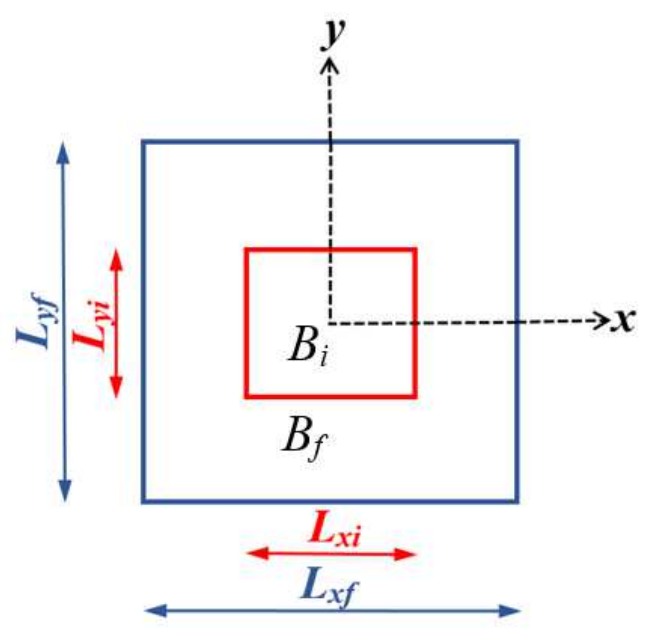

Fig. 8 Schematic representation of initial $\left(B_{i}\right)$ and pre-stretched $\left(B_{f}\right)$ continuum rectangular hyperelastic membrane.

expressed as a function of three principal stretches for the unit fibers that are initially oriented along the directions of three axes in the coordinate system, $\lambda_{1}, \lambda_{2}$, and $\lambda_{3}$, or in terms of the strain invariant $I_{1}, I_{2}$, and $I_{3}$ as

$$
\psi=\psi\left(\lambda_{1}, \lambda_{2}, \lambda_{3}\right)=\psi\left(I_{1}, I_{2}, I_{3}\right)
$$

\subsubsection{Constitutive modeling}

In this study, 3M VHB-4910 acrylic tape is the primary material used as the dielectric elastomer. Due to minimal changes in the volume of rubber-type material, incompressible nature is usually assumed, i.e., $I_{3}=1$, and the principal stretch in normal direction becomes $\lambda_{3}=\frac{1}{\lambda_{1} \lambda_{2}}$ or, physically, $\lambda_{3}=\frac{t_{f}}{t_{i}}$.

In the present study, the strain energy of $3 \mathrm{M}$ VHB-4910 acrylic tape is described using the neo-Hookean hyperelasticity material model from Eq. 12. In all the simulations, the shear modulus of the membrane is equated as $\mu=2 \mathrm{C}_{10}$, in which $C_{10}$ is the material parameter. The induced principal Cauchy stresses due to prestretching of the membrane for the neo-Hookean material model Soares and Gonçalves (2018) is expressed as 


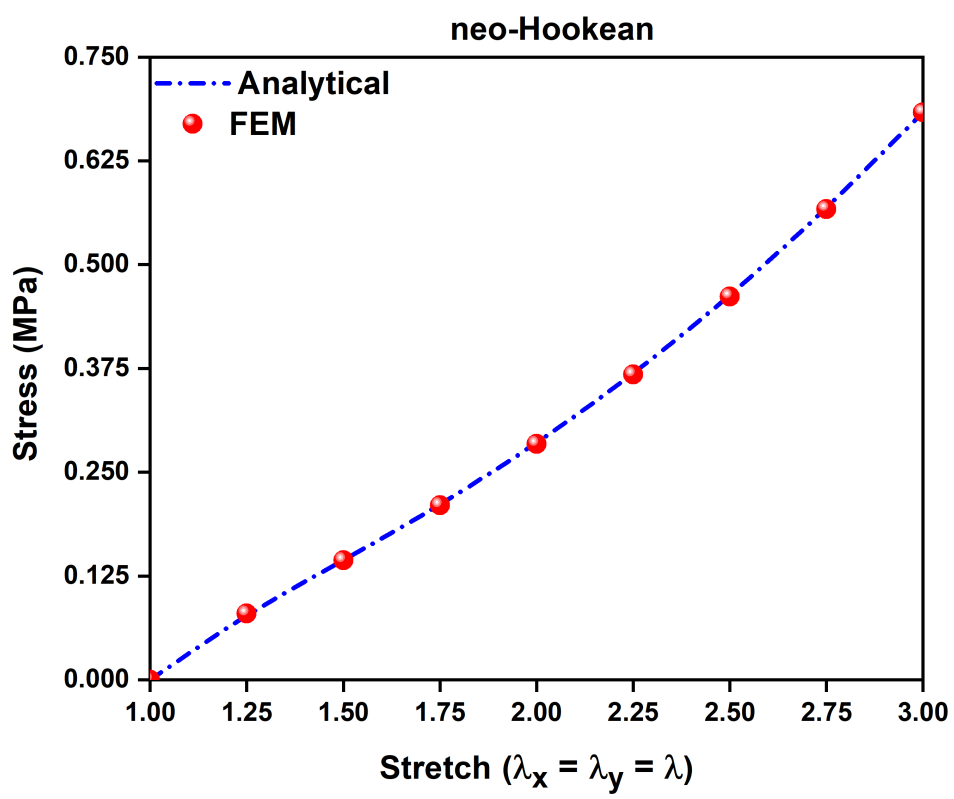

Fig. 9 An analytically validated stress-stretch curve of hyperelastic membrane using neo-Hookean model of hyperelasticity.

Table 2 Mechanical properties of the different parts of the DEMES implemented in ABAQUS.

\begin{tabular}{ccccc}
\hline Part & Material & Type & Young's modulus & Poisson's ratio \\
\hline Elastomer membrane & VHB 3M (4910) & Hyper-elastic & $E(298 K)=0.1537 \mathrm{MPa}$ Liu et al. (2015) & 0.499 \\
Frame & PET & Elastic & $5.7 \mathrm{Gpa}$ & 0.40 \\
Stiffeners & PLA & Elastic & $3.5 \mathrm{GPa}$ & 0.35
\end{tabular}

$$
\begin{aligned}
& \sigma_{1}=2 C_{10}\left(\lambda_{x}^{2}-\frac{1}{\lambda_{x}^{2} \lambda_{y}^{2}}\right) \\
& \sigma_{2}=2 C_{10}\left(\lambda_{y}^{2}-\frac{1}{\lambda_{x}^{2} \lambda_{y}^{2}}\right)
\end{aligned}
$$

\subsubsection{Numerical implementation}

Next, the numerical simulations using the finite element software ABAQUS 6.19, considering a hyperelastic membrane is performed. Convergence of the principal stresses of the membrane considering neo-Hookean material model up to four decimal places is evaluated by implementing the M3D4 mesh of 2500 elements. The obtained finite element results for the principal stresses agree well with the analytical results obtained using Eq. (19) (see Fig. 9). 
The flow process technique incorporated to measure the attained equilibrium angle of the DEMES using ABAQUS is revealed in Fig. 10. First of all, an initial hyperelastic membrane with M3D4 mesh element is considered, as depicted in Fig. 10 (a). An equal biaxial pre-stretch is provided to the considered membrane (Fig. 10(b)). The obtained principal stresses for different membrane stretches are considered as an initial condition of the membrane. Next, the prestretched DE membrane having the same dimensions as that of the compliant frame (Fig. 10(c)) is affixed on the frame surface to develop the assembly of the membrane and frame, as shown in Fig. 10(d). Finally, the 3-D printed PLA reinforcements and a stiffening frame made up of PET material is attached to the compliant frame to form the required DEMES, as shown in Fig. 10(e). The obtained deformed and undeformed configuration of the DEMES

Flow process implemented in ABAQUS

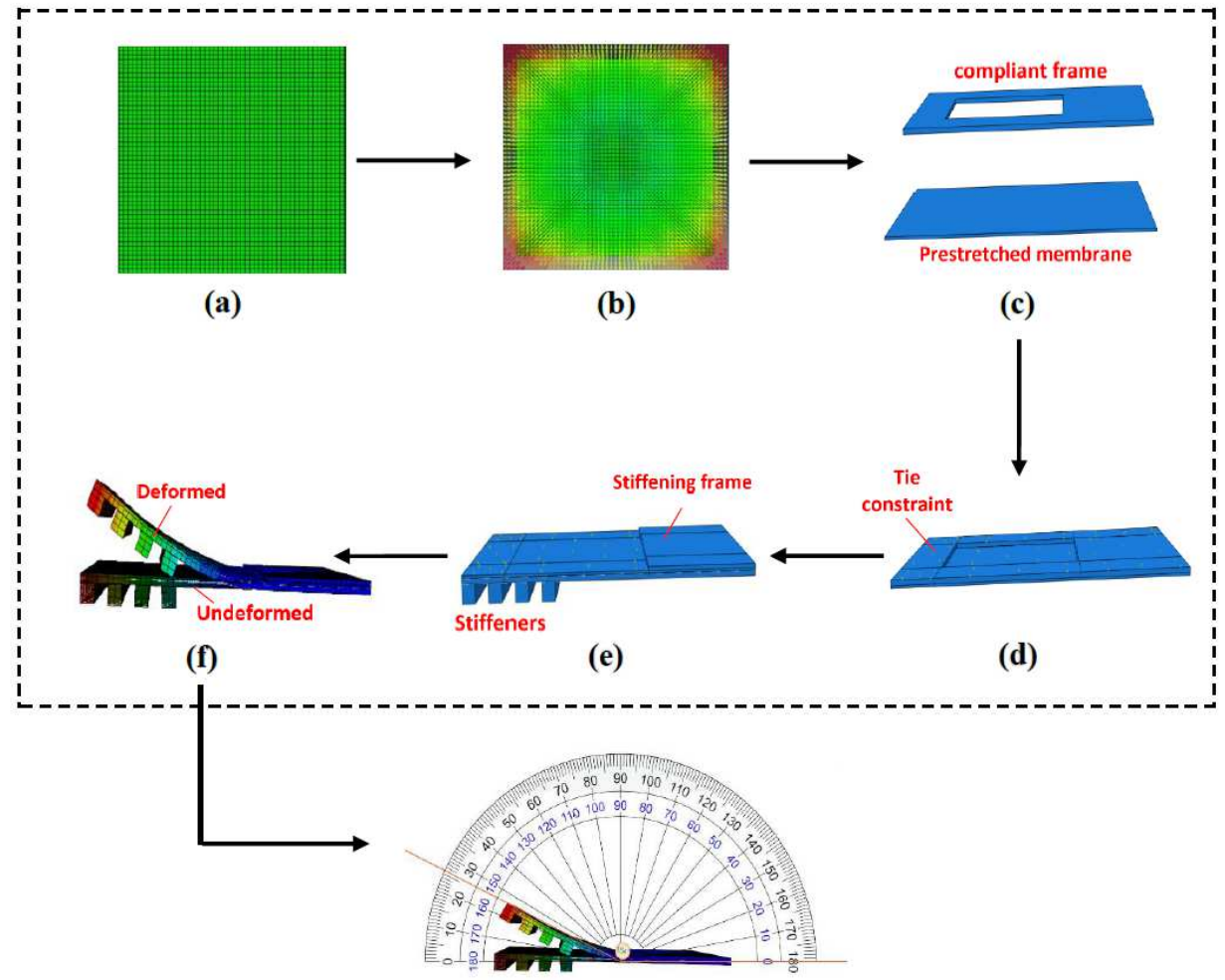

(g)

Fig. 10 Flow process representation implemented in ABAQUS to measure the attained equilibrium angle of the DEMES: (a) Initial meshed model of the hyperelastic membrane, (b) Biaxial pre-stretched membrane, (c) Compliant frame and pre-stretched membrane with same dimensions, (d) Assembly of the compliant frame and pre-stretched membrane, (e) Attached 3-D stiffeners and stiffening frame to the assembly, (f) Deformed and undeformed shape of the DEMES, (g) Measurement of equilibrium angle using a commercially available online protector. 
is depicted in Fig. 10(f). Finally, the equilibrium angle of the DEMES is obtained by using a commercially available online protector (Fig. 10(g)). The material properties of the elastomeric membrane, inextensible PET frame, and 3D printed PLA reinforcements considered for numerical simulations are indexed in Table 2.

\subsection{Validation of the proposed model}

To affirm the executability of the proposed static analytical framework, we have enclosed a comparison of the obtained analytical model results with performed experimental and numerical studies. Figure 11 presents this comparison with the experimental and numerical observations on a configurationally similar prototype of DEMES for four different prestretch levels $\lambda_{p}=1.25,1.5,1.75,2.0$ with $S=0.5 \mathrm{~cm}$, and $b=1.5 \mathrm{~cm}$, respectively .

The predictions are made for the attained equilibrium angle $\left(\theta_{e q}\right)$ of the DEMES when it is detached from external mechanical constraints. The examination demonstrates the enlargement in value of the equilibrium angle with increasing prestretch $\left(\lambda_{p}\right)$ of the membrane. The present static model effectively captures the deviation of the equilibrium angle with applied prestretch as observed in the experimental and numerical observations. To get the accurate experimental predictions of the equilibrium angle of the DEMES, we conducted three sets of experiments for all the specified prestretch levels. Figure 11 shows the upper and lower deviations in $\theta_{e q}$ obtained experimentally for all considered prestretch levels. From Fig. 11, it is also observed

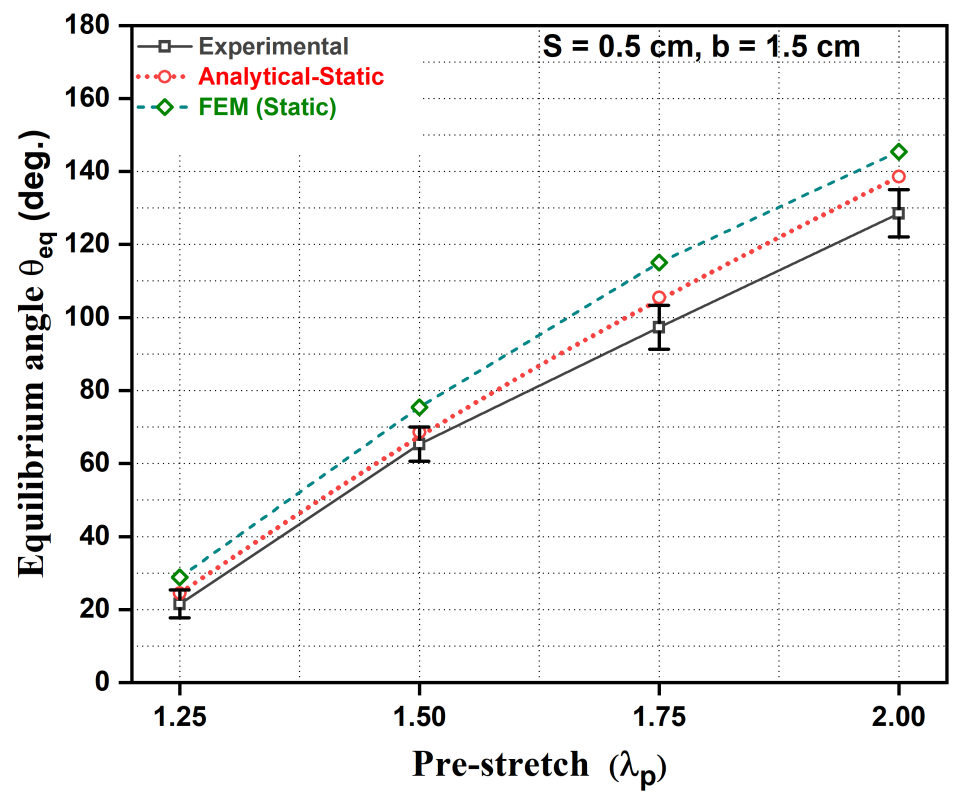

Fig. 11 Experimental and numerical validation of the proposed analytical framework. 
that the standard deviation in the measurement of $\theta_{e q}$ obtained experimentally increases with the increase in the prestretch levels. These large experimental deviations in equilibrium angle measurement can be attributed to fabrication variations. Further, the seemingly slight discrepancy in the attained equilibrium angle in the developed analytical and numerical model with the experimental observations arises due to the inherent viscoelastic nature of the VHB-4910, which is not considered in the present analytical and numerical modeling of the DEMES. However, Fig. 11 shows an excellent corroboration between the upper deviations of the experimental predictions and those extracted using the developed static model, hence ascertaining the legitimacy of the developed model.

As the proposed static analytical framework effectively predicts the attained equilibrium angle of the DEMES; we further extend the analytical framework in the upcoming section to analyze the nonlinear dynamic behavior of the proposed DEMES.

\section{Dynamic analysis of DE based minimum energy structures}

This section pertains to the development of a nonlinear dynamic model of the DEMES to investigate the effect of various geometrical and mechanical parameters along with incorporated stiffeners on the attained initial and final configuration of the DEMES.

\subsection{Dynamic governing equations}

The nonlinear dynamic governing equation of the developed DEMES is evaluated by using the Euler-Lagrange's (E-L) equation of motion based on the least action principle and is expressed as

$$
\frac{d}{d t}\left(\frac{\partial L}{\partial \dot{\theta}}\right)-\frac{\partial L}{\partial \theta}=0,
$$

in which $\dot{\theta}$ denotes the derivative of the bending angle with time and $\mathscr{L}$ signifies the Lagrangian, which is expressed as the difference between the total kinetic and potential energy of the DEMES. In the following subsection, we devise the expression of the total kinetic energy of the developed DEMES.

\subsection{Total kinetic energy $(K)$ of DEMES}

The considered structure comprises of a compliant frame with 3-D stiffeners embedded on its surface. This assembly of 3-D stiffeners, and the compliant frame adheres to a prestretched DE membrane whose weight is negligible. Hence, we ignore the effect of DE membrane weight in comparison to the compliant frame and stiffeners. Further, we consider the compliant frame as a rotational joint to evaluate the total kinetic energy of the developed DEMES as 


$$
K=\underbrace{\frac{m_{f} C^{2}}{24}\left(\frac{d \theta}{d t}\right)^{2}}_{\text {Frame }}+\underbrace{\frac{1}{2} m_{s} \sum_{n=1}^{N} V_{n}^{2}}_{\text {Stiffeners }}
$$

where, $m_{f}$ and $m_{s}$ represent the mass of the compliant frame and 3-D stiffener, respectively.

To obtain the final expression for the kinetic energy $(K)$ of the DEMES, the resultant velocities $\left(V_{1}-V_{4}\right)$ associated with the incorporated $(N=4)$ stiffeners need to be evaluated. The methodology implemented to determine the velocities are indexed in Appendix A. Upon invoking the expressions of the velocities from the Appendix A, the final kinetic energy of the developed DEMES ( $N=4$ stiffeners) is written as

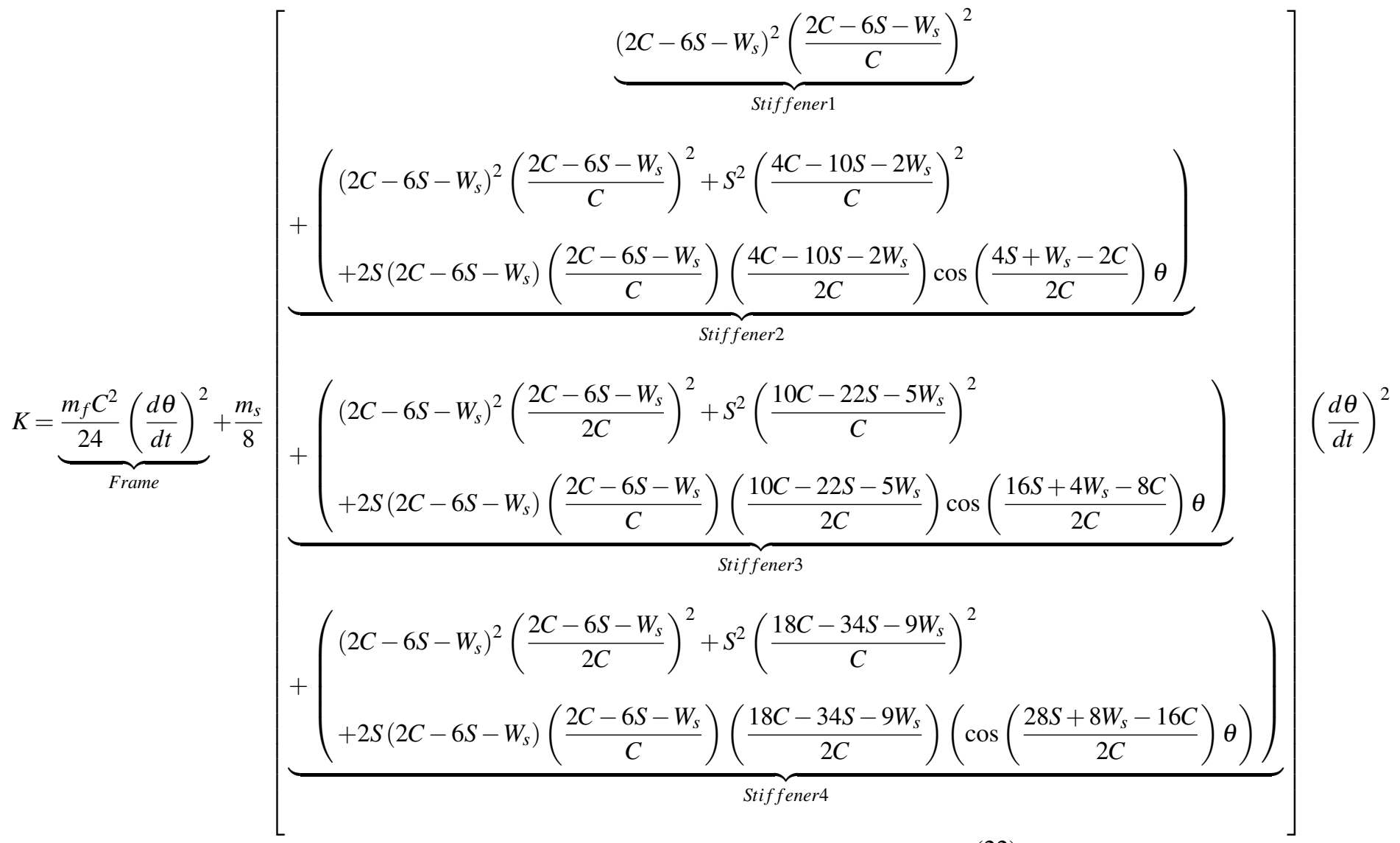

By invoking the expressions of total kinetic energy $(K)$ and total potential energy $(U)$ of the DEMES from Eq. (22) and Eq. (17) in Lagrange's equation of motion (Eq. 20 ), the ensuing nonlinear dynamic equation that governs the motion of developed DEMES is expressed as 


$$
\frac{m_{f} C^{2}}{12}\left(\frac{d^{2} \theta}{d t^{2}}\right)+\frac{m_{s}}{4}\left(Z_{1}\right)\left(\frac{d^{2} \theta}{d t^{2}}\right)-\frac{m_{s}}{8}\left(Z_{2}\right)\left(\frac{d \theta}{d t}\right)^{2}+\left(Z_{3}\right) \theta+\mu W_{0} L_{0} t_{0}\left(Z_{4}\right)=0
$$

The required expressions for the terms $Z_{1}-Z_{4}$ are provided in the Appendix B for the ready reference.

Initially, when a pre-stretched membrane is attached to the compliant frame, the structure remains flat. Hence, the associated two initial condition that correspond to the developed DEMES are

$$
\left.\theta\right|_{t=0}=0 ;\left.\quad \frac{d \theta}{d t}\right|_{t=0}=0
$$

The dynamic behavior of the developed DEMES is evaluated by simulating the governing differential Eq. (23) along with initial conditions from Eq. (24) using the built-in MATLAB ODE45 solver. Specifically, ODE45 solver incorporates the $4^{\text {th }}$ order Runge Kutta method to solve the differential equations with truncation error of order $d t^{5}$, in which $d t$ represents the time step. In the next section, the influence of incorporated stiffeners on the attained initial and final configurations of the DEMES are investigated.

\section{Results and analysis}

The present section investigates the effect of different geometrical parameters on the nonlinear dynamic behavior of the proposed DEMES. To interrogate the effect of stiffeners implemented on the surface of the frame, we vary the spacing $S=0.25$, $0.50,0.75$, and $1.0 \mathrm{~cm}$ between the adjacent stiffeners. The bending stiffness of the frame is also tuned by varying the levels of the width of bending strips, i.e., $b=1.0$, $1.5,2.0,2.5$, and $3.0 \mathrm{~cm}$. Further, four different levels of equal bi-axial prestretch $\lambda_{p}$ $=1.25,1.50,1.75$, and 2.0 of the DE membrane is implemented. The shear modulus $(\mu)$ of the DE membrane obtained at room temperature $(T=298 \mathrm{~K})$ is considered Liu et al. (2015) for all the simulations.

In the following, we exhibit a parametric investigation to examine the influence of prestretch $\left(\lambda_{p}\right)$ of the membrane, spacing $(S)$ between the stiffeners, width $(b)$ of the bending strips of the frame on the obtained equilibrium angle of the structure when it is disengaged from external mechanical constraints.

\subsection{Attainment of the initial equilibrium configuration}

The equilibrium state attained by the structure can be tuned by altering the various geometrical and material properties of the membrane, frame, and stiffeners incorporated to develop the proposed DEMES. In this subsection, we carried out a parametric study to assess the impact of prestretch $\left(\lambda_{p}\right)$ of the membrane, bending strips width $(b)$ of the frame, and spacing $(S)$ between the stiffeners on the attained initial equilibrium state of the DEMES. The DEMES actuator attained an equilibrium configuration 


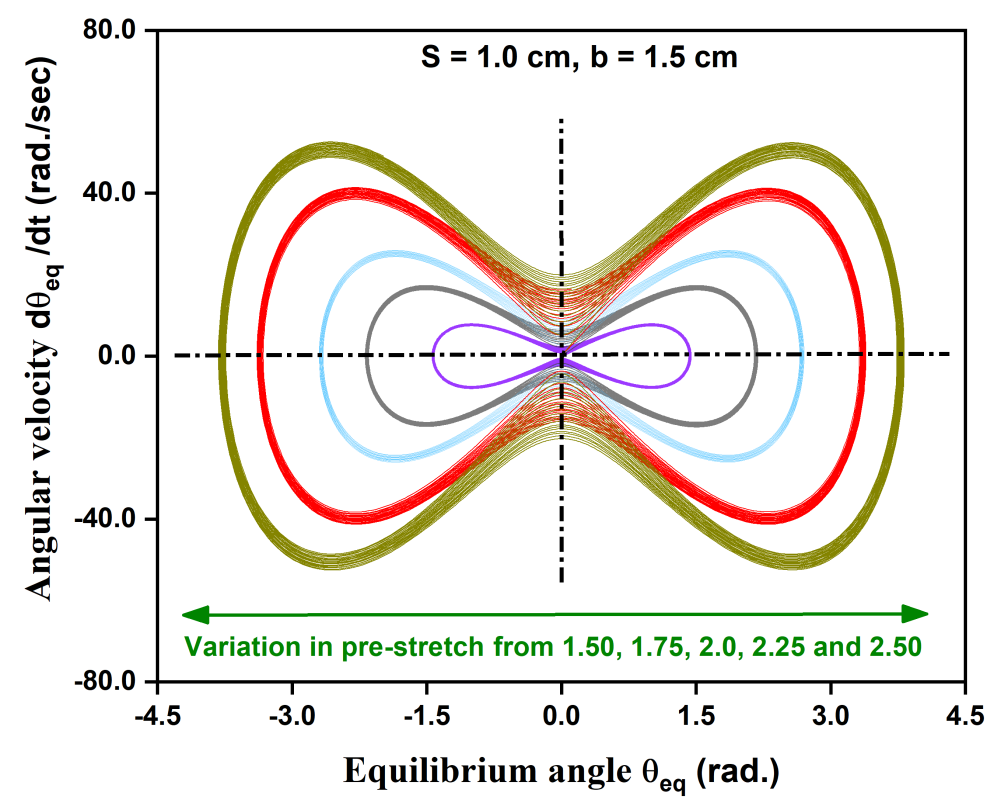

Fig. 12 Effect of prestretch $\left(\lambda_{p}\right)$ of the DE membrane on the equilibrium angle $\left(\theta_{e q}\right)$ of the DEMES.

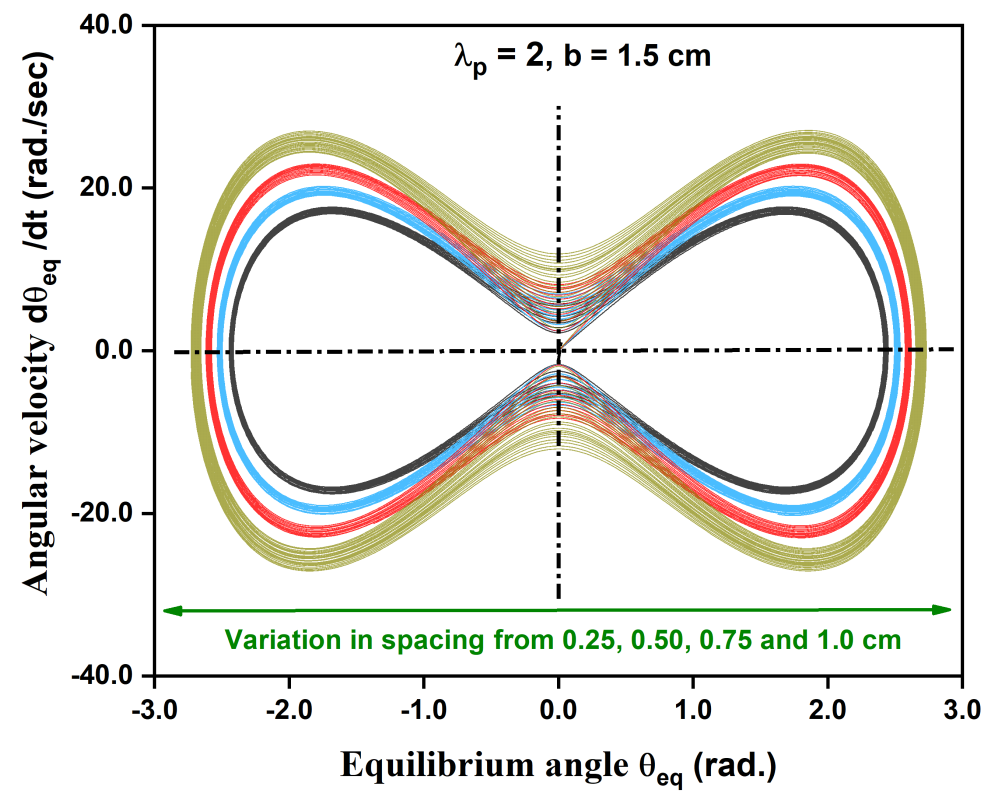

Fig. 13 Effect of spacing $(S)$ between the adjacent stiffeners on the equilibrium angle $\left(\theta_{e q}\right)$ of the DEMES. 
due to redistribution of energy between the elastomer film and the inextensible frame. Thus, no electrical load $(E=0)$ is applied for the cases considered in this subsection.

First of all, we evaluate the effect of prestretch $\left(\lambda_{p}\right)$ of the membrane on the attained equilibrium angle $\left(\theta_{e q}\right)$ of the DEMES. For this purpose, we obtain the phase portraits illustrating the alteration of equilibrium angle $\left(\theta_{e q}\right)$ with angular velocity $\left(\frac{d \theta_{e q}}{d t}\right)$ for different prestrech level $\left(\lambda_{p}=1.50,1.75,2.0,2.25\right.$, and 2.50$)$ at specified conditions of $S=1.0 \mathrm{~cm}$, and $b=1.5 \mathrm{~cm}$, respectively as shown in Fig. 12. From Fig. 12 , it is inferred that the structure for all the considered prestretch levels manifests a supercritical pitchfork bifurcation of equilibrium configuration Khurana et al. (2020); Strogatz (2018). The stated bifurcation of equilibrium configuration represents the attainment of positive as well as negative equilibrium angle of the equal magnitude. This bifurcation of the equilibrium state is referred to as the bi-stability of DEMES Follador et al. (2015); Zhao et al. (2016a).

Next, the phase portraits to investigate the influence of spacing $(S)$ between the adjacent stiffeners on the attained equilibrium angle of the structure for the aforementioned four spacing levels is plotted (see Fig. 13). This figure reveals that the attained $\theta_{e q}$ of the DEMES amplifies as the spacing $(S)$ between the adjacent stiffeners increases for the specified conditions. This is because as spacing $(S)$ increases, the bending moment imposed by the stiffeners decreases. Additionally, Fig. 13 also shows that the envelope illustrating the maximum and minimum value of angular velocity $\left(\frac{d \theta_{e q}}{d t}\right)$ enlarges as spacing $(S)$ between the adjacent stiffeners increases.

The developed model of DEMES incorporates a compliant frame with two bending strips having a width $(b / 2)$ each (see Fig. 3 a). The total width $(b)$ of the bending strips alters the bending stiffness of the compliant frame. From Fig. 14 a, it is observed that increase in width $(b)$ of the frame enhances the bending stiffness of the compliant frame. Due to this increase in bending stiffness with the increase in width (b), the frame becomes more restrictive to the bending moment imposed by the DE membrane. Hence, the attained equilibrium angle $\left(\theta_{e q}\right)$ of the structure decreases with the enhancement in width $(b)$ of the bending strips. It is inferred that tuning of both prestretch $\left(\lambda_{p}\right)$ of the membrane and bending strips width $(b)$ of the compliant frame alters the equilibrium state of the structures. Therefore, to investigate the combined effect of $\lambda_{p}$ and $b$, we obtained $\theta_{e q}$ of the structure for three different prestretch level $\lambda_{p}=1.50,2.0$, and 2.50 with aforementioned varying bending strips width $b$ as depicted in Fig. 14 b. As observed from Fig. 14 b, the DEMES with a larger value of $\lambda_{p}$ and a smaller value of $b$ attains the maximum value of $\theta_{e q}$. This is because the compliant frame offers less bending stiffness at a lower value of $b$ and membrane possess more strain energy at a higher value of $\lambda_{p}$, which causes the more bending of the structure and hence enhances the equilibrium angle of the structure.

Further, we study the combined influence of spacing $(S)$ between the adjacent stiffeners and prestretch $\left(\lambda_{p}\right)$ of the membrane. From Fig. 15, it is visualized that the structure with higher spacing $(S)$ between the stiffeners and higher membrane prestretch $\left(\lambda_{p}\right)$ exhibit the highest value of $\theta_{e q}$. The enhancement in the spacing $(S)$ reduces the bending moment posed by the stiffeners and more prestretching of the membrane enhances the membrane strain energy and thereby increases the attained 


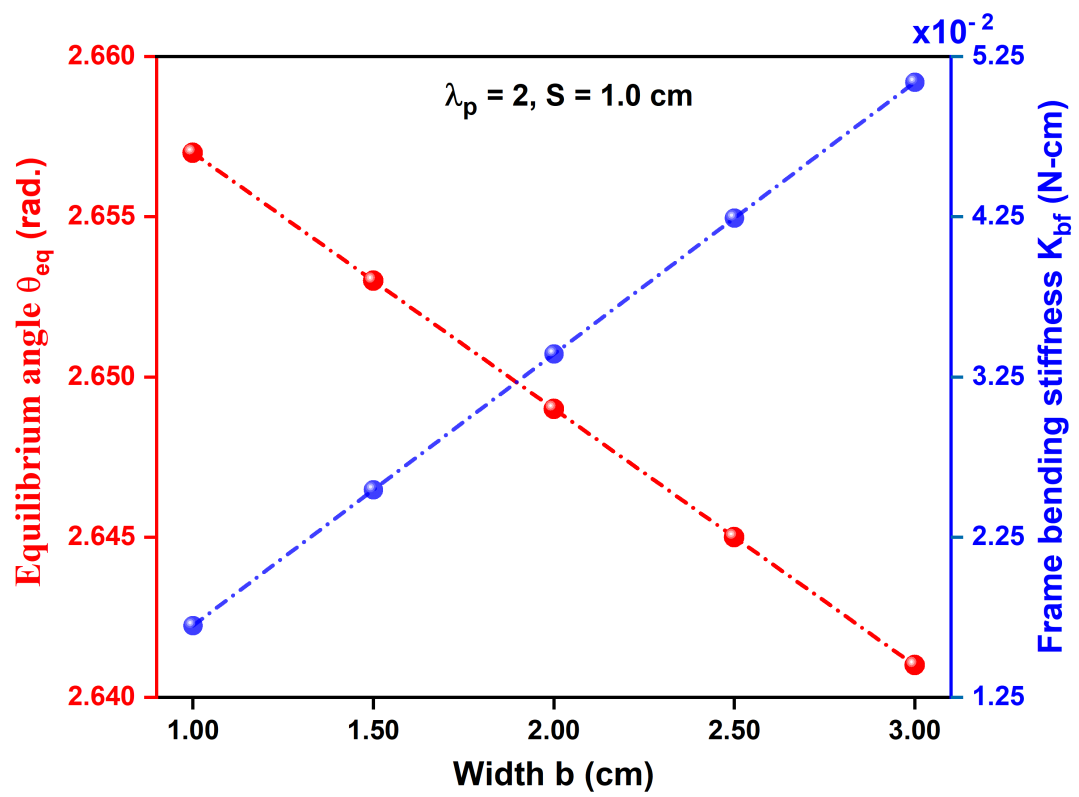

(a)

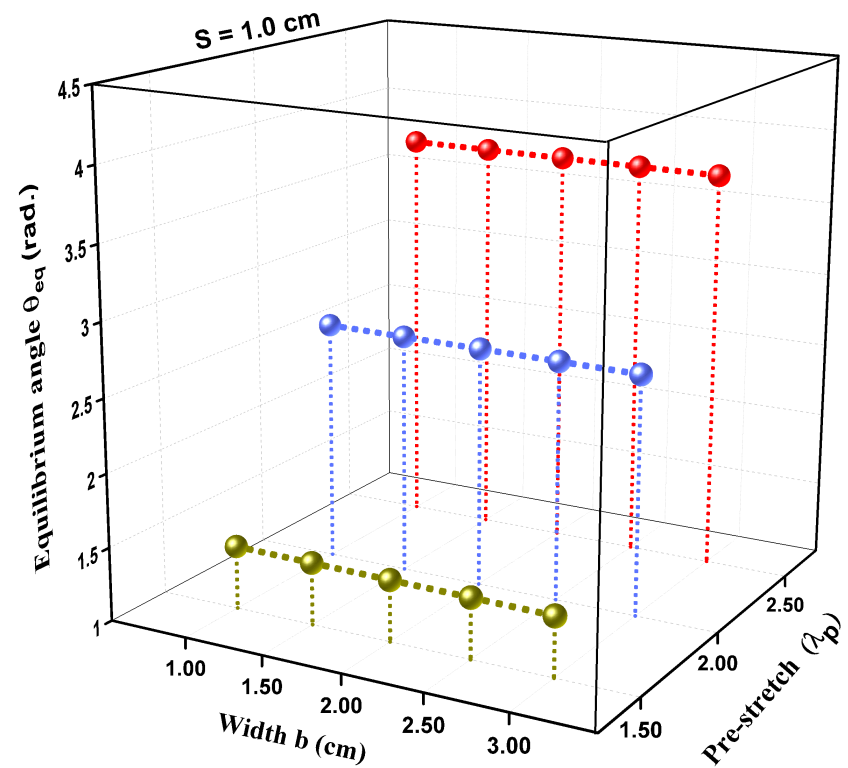

(b)

Fig. 14 Variation of (a) Equilibrium angle $\left(\theta_{e q}\right)$ and bending stiffness of the frame with width $b$ of the bending strips and (b) Combined effect of width $b$ of the bending strips and prestretch of the membrane on $\theta_{e q}$. 


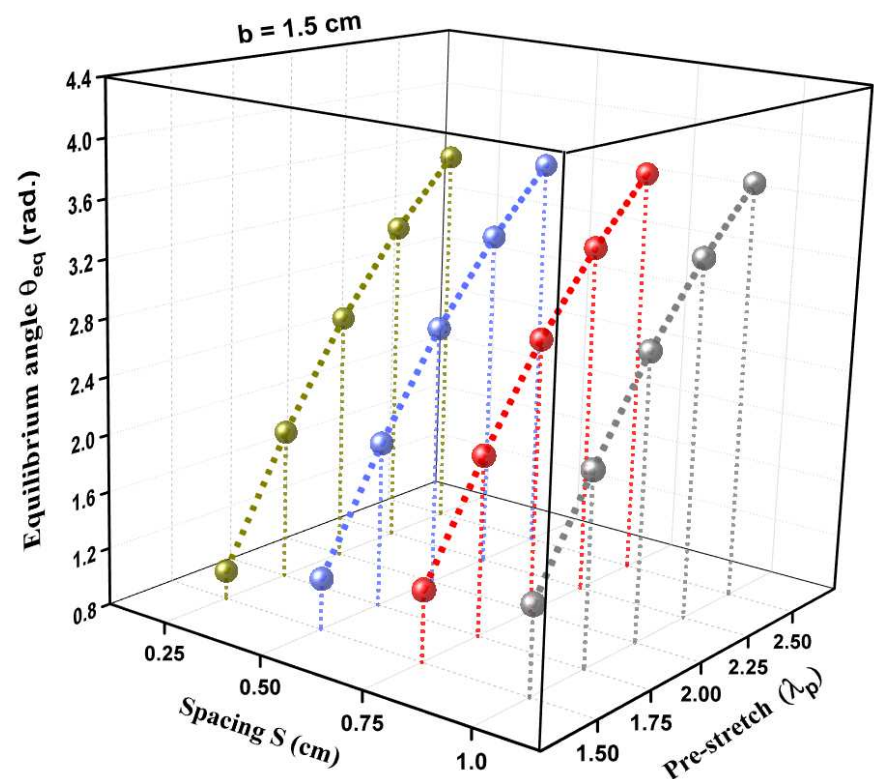

Fig. 15 Combined effect of spacing $(S)$ between the adjacent stiffeners and prestretch $\left(\lambda_{p}\right)$ of the membrane on $\theta_{e q}$.

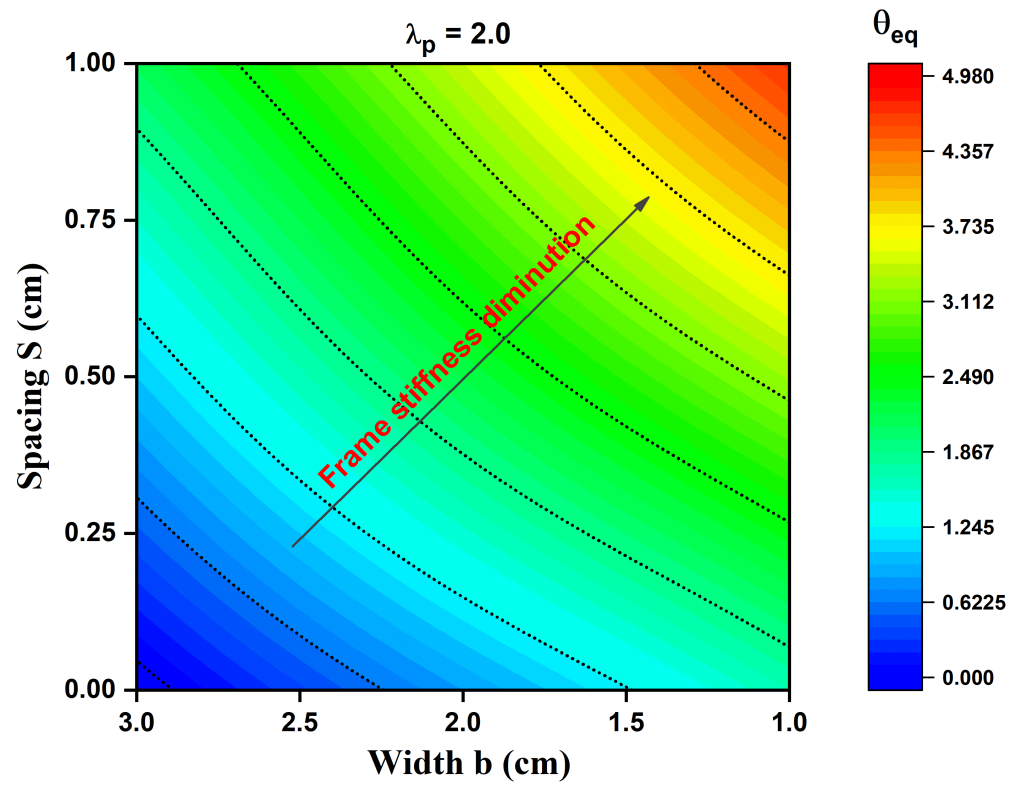

Fig. 16 Effect of bending strips width $(b)$ of the compliant frame and spacing $(S)$ between the adjacent stiffeners on the equilibrium angle $\left(\theta_{e q}\right)$ of the DEMES. 
$\theta_{e q}$ of the structure. Finally, the combined influence of bending strips width $(b)$ and spacing $(S)$ between the adjacent stiffeners on the attained equilibrium angle $\left(\theta_{e q}\right)$ of the structure is investigated by contour plots (Fig. 16). From Fig. 16, it is inferred that the DEMES exhibits the maximum value of $\theta_{e q}$ at a lower level of bending strips width $(b)$ and at a higher level of spacing $(S)$ between the adjacent stiffeners. The justification for this enhancement in the $\theta_{e q}$ of the DEMES is due to the diminution of the compliant frame stiffness at a lower value of $b$ and at higher spacing $(S)$ between the adjacent stiffeners. Due to this reduction in the bending stiffness, less resistance is offered by the frame against the bending moment posed by the membrane and thereby enhances the structure's equilibrium angle $\left(\theta_{e q}\right)$.

\subsection{DC dynamic response}

In this subsection, we consider the obtained values of equilibrium angle $\theta_{e q}$ of the DEMES in the preceding section as the initial conditions for interrogating the DC dynamic behavior of the structure. A time- independent electric field (e) approximately represented by a constant Heaviside step function is applied. The applied electric field results in the periodic oscillations of the structure, whose time and intensity rely on the magnitude of applied DC voltage signal $\left(V_{d c}\right)$.

The DEMES actuator with initial $\theta_{e q}$ corresponding to the conditions $\lambda_{p}=2.0$, $S=1.0, \mathrm{~cm}$ and $b=1.5 \mathrm{~cm}$ is excited by the DC voltage signal up to the threshold state at which the structure almost becomes flat, i.e., the tip angle practically becomes equal to zero as shown in Fig. 17 a. The tip angle of the DEMES is denoted by $\theta_{t}$, and is defined as: $\left\{\begin{array}{l}\left.\theta_{t}\right|_{V_{d c}=0}=\theta_{e q} \\ \left.\theta_{t}\right|_{V_{d c} \neq 0}=\theta\end{array}\right\}$. An appreciable reduction in the tip angle of the DEMES with the increase in applied DC voltage is perceived, as shown in Fig. 17 a. Next, the actuation performance characterized by the actuation range $\left(\theta_{e q^{-}} \theta\right)$ of the DEMES actuator for different levels of spacing $(S)$ between the adjacent stiffeners corresponding to $V_{d c}=4000 \mathrm{~V}, \lambda_{p}=2.0, S=1.0 \mathrm{~cm}$ and $b=1.5 \mathrm{~cm}$, respectively, is plotted in Fig. 17 b. From the figure, it is inferred that the enhancement in the spacing $(S)$ has a favorable impact on the actuation range of the DEMES actuator. This is because the DEMES with higher spacing $(S)$ between the adjacent stiffeners possess the lower value of frame stiffness resulting in more bending of the structure at applied particular DC voltage, thereby enhancing the actuation range of the DEMES actuator.

Next, the phase portraits revealing the alteration of angular velocity $\left(\frac{d \theta}{d t}\right)$ with bending angle $(\theta)$ is plotted for varying levels of applied DC dynamic voltage (Fig. 18 a) and different spacing $(S)$ between the adjacent stiffeners (Fig. 18 b). As anticipated, the bending angle $(\theta)$ of the DEMES decreases as applied DC voltage increases, as shown in Fig. 18 a. Further, the envelope of the phase portraits (Fig. 18 a) enhances with the depletion of the DC dynamic voltage. From Fig. $18 \mathrm{~b}$, it is visualized that the bending angle $(\theta)$ of the DEMES amplifies as the level of spacing $(S)$ between the adjacent stiffeners increases. Further, the envelope of the phase portraits (Fig. 18 b) enlarges with the increase in spacing $(S)$ between the adjacent stiffeners. Finally, the combined effect of applied DC dynamic voltage and spacing $(S)$ on the tip angle $\left(\theta_{t}\right)$ of the structure is evaluated as depicted in Fig. 19. From Fig. 19, the maximum 


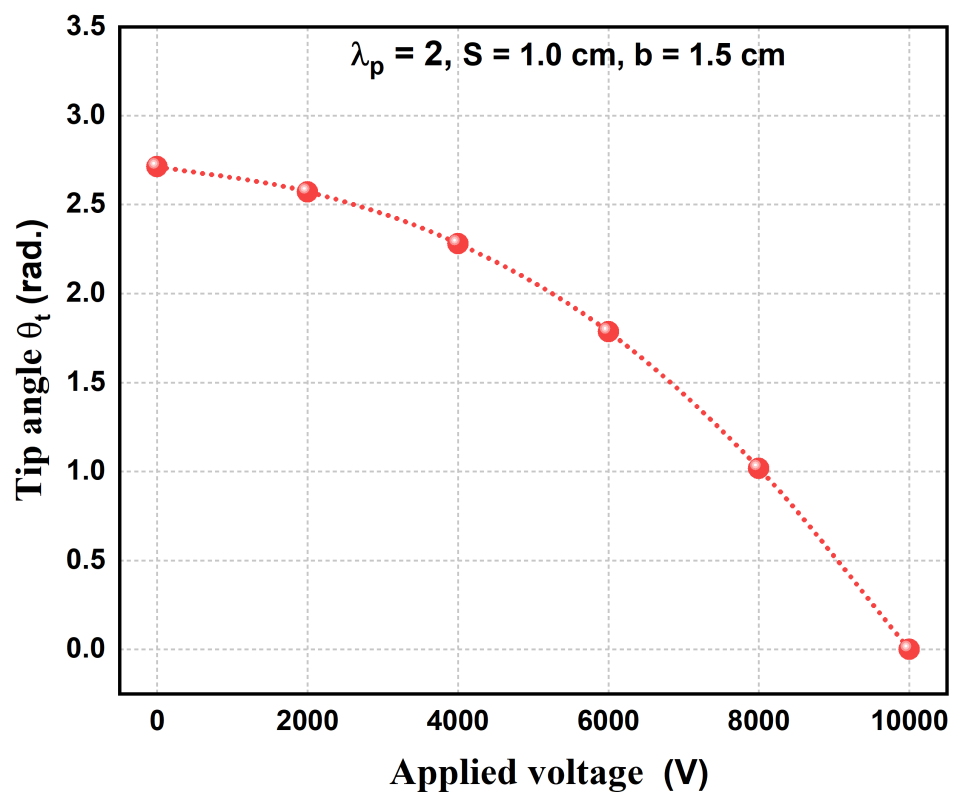

(a)

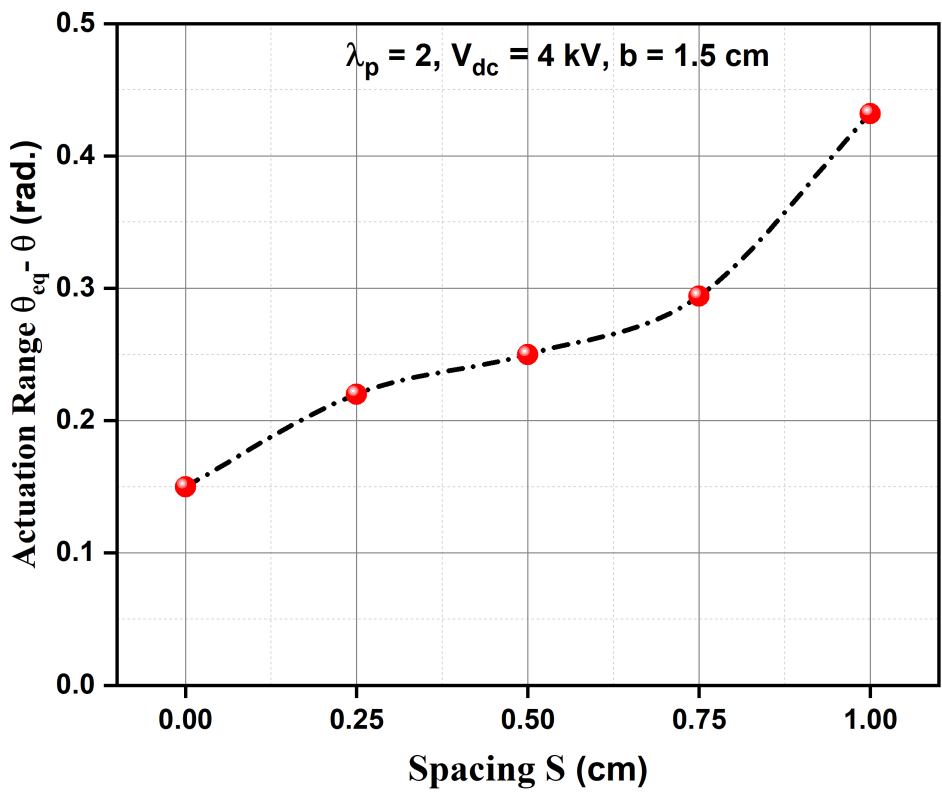

(b)

Fig. 17 DC dynamic behavior of the DEMES actuator showing the (a) Alteration of tip angle with applied DC voltage, (b) Actuation range $\left(\theta_{e q^{-}} \theta\right)$ for different spacing between the adjacent stiffeners at a specified applied voltage level of $V_{d c}=4 \mathrm{kV}$. 


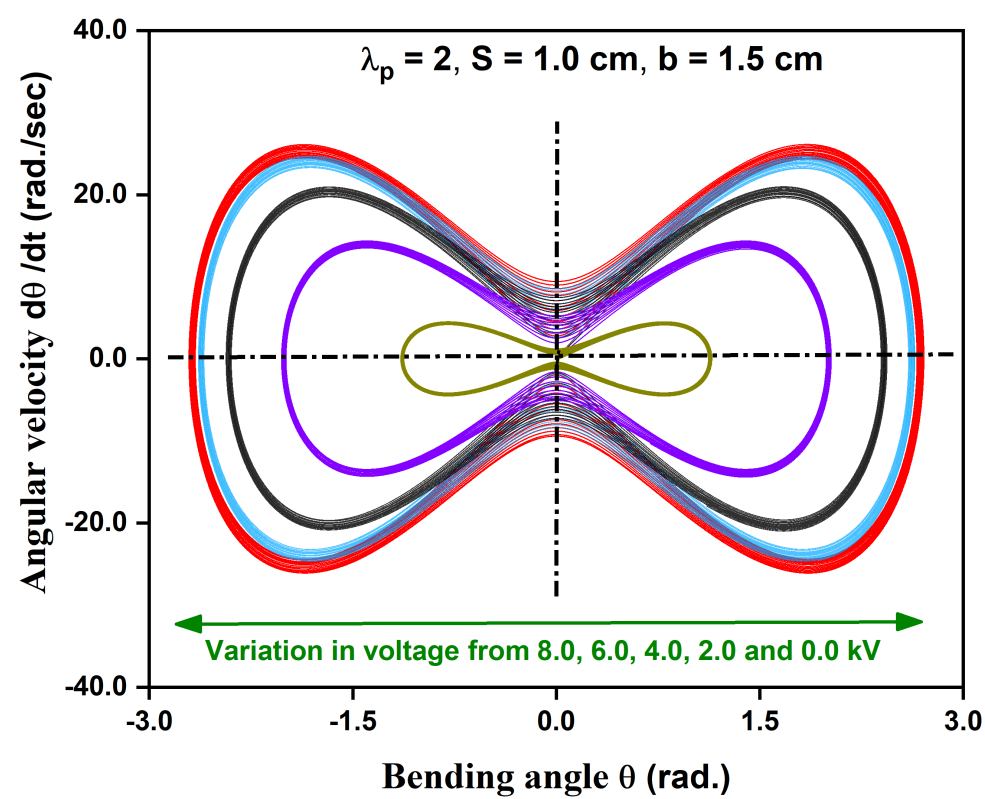

(a)

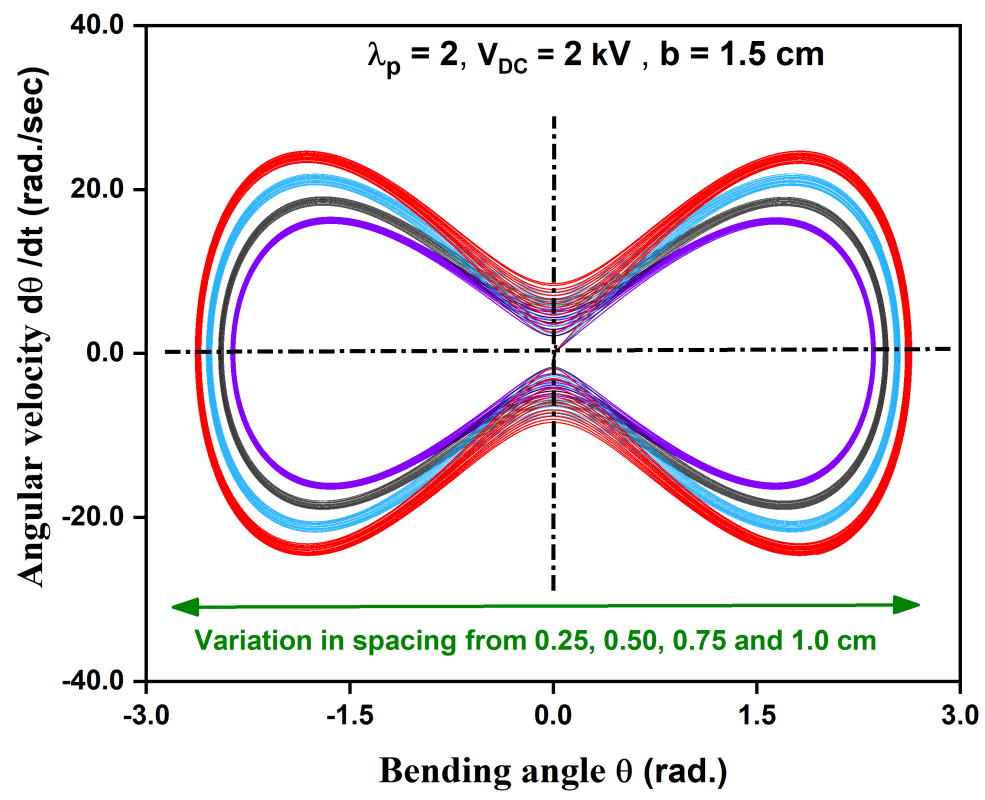

(b)

Fig. 18 Phase portraits showing the variation of angular velocity $\left(\frac{d \theta}{d t}\right)$ with bending angle $(\theta)$ for (a) different levels of applied DC voltage, (b) different levels of spacing $(S)$ between the consecutive stiffeners. 


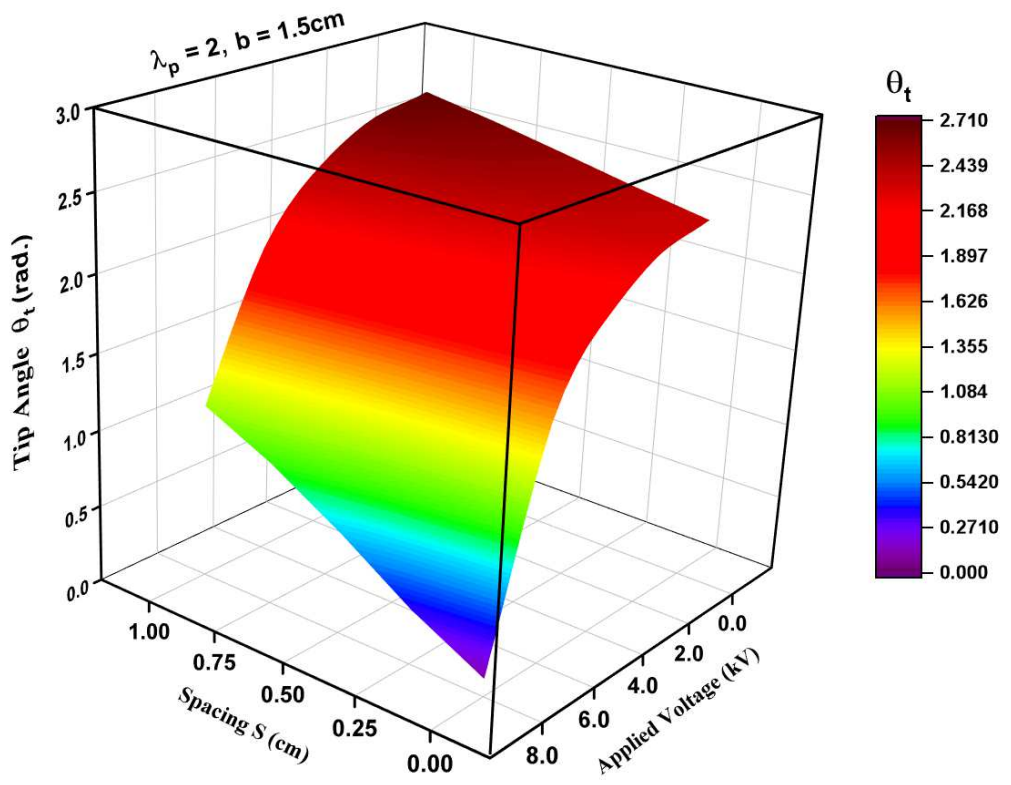

Fig. 19 Combined effect of applied DC dynamic voltage and spacing $(S)$ on the tip angle of the DEMES.

reduction in the tip angle of the DEMES is observed at a lower spacing level and at a higher level of applied DC voltage.

\section{Concluding remarks}

In summary, this work investigated the role of stiffeners in enhancing the electromechanical behavior of dielectric elastomer minimum energy structures. The primary motive of adding stiffeners is to circumvent the warping resulting from anticlastic curvature formed in the elementary rectangular DEMES. The effect of including stiffeners is assessed from two perspectives; they are: (a) impact on the initial equilibrium angle of the DEMES prior to electromechanical actuation, and (b) impact on the electromechanical response of the DEMES when driven by a Heaviside electric load. To this end, an energy-based analytical model is devised to predict the initial equilibrium angle as a function of the underlying geometric and material parameters. The predictive capabilities of the proposed model are corroborated with the 3D finite element simulations and the experimental observations. The analytical model is then extended to accommodate the time-dependent effects for assessing the nonlinear dynamic response of DEMES driven by the electric load.

In particular, we investigated the effect of (i) levels of pre-stretch, (ii) width of the bending strip, (iii) spacing between the stiffeners, and (iv) applied voltage on the initial equilibrium angle and the angular stroke resulting from the electromechanical actuation. The initial equilibrium angle attained by the DEMES increases with 
increasing levels of pre-stretch on virtue of the fact that increasing amount of strain energy is available for bending the compliant frame. On parallel lines, for a fixed number of stiffeners, the initial equilibrium angle is found to increase with increasing spacing between the stiffeners; whereas it reduces with increasing frame-width. With electric load used to drive the DEMES actuator, an appreciable diminution in the tip angle of the DEMES with an enhancement in the applied voltage is observed. The actuation range of the DEMES actuator for maximum considered spacing $(S=1 \mathrm{~cm})$ between the adjacent stiffeners is enhanced by $28.2 \%$ with respect to the DEMES actuator with no spacing $(S=0 \mathrm{~cm})$ at a particular level of applied DC voltage. This clearly indicates the dependency of various geometric and operating parameters on the actuation performance of the DEMES actuator.

The analytical model presented in this work can be of potential use in predesigning a DEMES actuator deprived of warping and can further be refined to accommodate additional effects of practical relevance, such as dissipative material models, (e.g, viscoelasticity) and complex loading conditions (e.g., AC electric loading).

\section{Acknowledgement}

This research is supported financially by the Science and Engineering Research Board (SERB), India through Grant No. EMR/2017/003289

\section{Data availability}

The authors declare that data supporting the findings of this study are available within the article.

\section{Declarations}

Conflict of interest: The authors declared no potential conflicts of interest with respect to the research, authorship, and/or publication of this article.

\section{Appendix A: Evaluation of velocities} The associated curved lengths and angles based on the embedded stiffeners on the
surface of the compliant frame is obtained geometrically by using Fig. $3 \mathrm{c}$ as 


$$
\begin{aligned}
& \psi_{1}=\theta_{1}+\left(\frac{\theta_{2}-\theta_{1}}{2}\right)=\left(\frac{\theta_{1}+\theta_{2}}{2}\right), \psi_{2}=\psi_{1}+\left(\frac{\theta_{3}+\theta_{4}}{2}\right)=\left(\frac{\theta_{1}+\theta_{2}+\theta_{3}+\theta_{4}}{2}\right) \\
& \psi_{3}=\psi_{2}+\left(\frac{\theta_{5}+\theta_{6}}{2}\right)=\left(\frac{\theta_{1}+\theta_{2}+\theta_{3}+\theta_{4}+\theta_{5}+\theta_{6}}{2}\right) \\
& \psi_{4}=\psi_{3}+\left(\frac{\theta_{7}+\theta_{8}}{2}\right)=\left(\frac{\theta_{1}+\theta_{2}+\theta_{3}+\theta_{4}+\theta_{5}+\theta_{6}+\theta_{7}+\theta_{8}}{2}\right)
\end{aligned}
$$

$$
\begin{aligned}
& \theta_{1}=\left(\frac{C_{1}}{C}\right) \theta, \theta_{2}=\left(\frac{C_{2}}{C}\right) \theta, \theta_{3}=\left(\frac{C_{3}}{C}\right) \theta, \theta_{4}=\left(\frac{C_{4}}{C}\right) \theta \\
& \theta_{5}=\left(\frac{C_{5}}{C}\right) \theta, \theta_{6}=\left(\frac{C_{6}}{C}\right) \theta, \theta_{7}=\left(\frac{C_{7}}{C}\right) \theta, \theta_{8}=\left(\frac{C_{8}}{C}\right) \theta=\left(\frac{C}{C}\right) \theta=\theta
\end{aligned}
$$

$$
\frac{d x_{1}}{d t}=\frac{d x_{1}}{d \theta} \times \frac{d \theta}{d t}=\left(C-3 S-\frac{W_{s}}{2}\right)\left(-\sin \left(\frac{C_{1}+C_{2}}{2 C}\right) \theta\right)\left(\frac{C_{1}+C_{2}}{2 C}\right) \frac{d \theta}{d t}
$$

The curved lengths associated with the incorporated stiffeners depicts in Fig. $3 \mathrm{c}$ are obtained as

$$
\begin{aligned}
& C_{1}=C-3 S-W_{s}, C_{2}=C-3 S, C_{3}=C-2 S-W_{s}, C_{4}=C-2 S \\
& C_{5}=C-S-W_{s}, C_{6}=C-S, C_{7}=C-W_{s}, C_{8} \approx \mathrm{C}
\end{aligned}
$$

To estimate the expression for the total kinetic energy of the DEMES (Eq. (21)), we derived the associated expressions for the velocities associated with the incorporated $(N=4)$ stiffeners. The expression for velocity $V_{1}$ is obtained as

$$
V_{1}^{2}=\left(\frac{d x_{1}}{d t}\right)^{2}+\left(\frac{d y_{1}}{d t}\right)^{2}
$$

Where,

$$
x_{1}=\left(C_{1}+\frac{W_{8}}{2}\right) \cos \psi_{1}, y_{1}=\left(C_{1}+\frac{W_{8}}{2}\right) \sin \psi_{1}
$$

On substituting the expression of $\psi_{1}$ from Eq. (A.1) in Eq. (A.5), we get

$x_{1}=\left(C-3 S-\frac{W_{s}}{2}\right) \cos \left(\frac{\theta_{1}+\theta_{2}}{2}\right)=\left(C-3 S-\frac{W_{s}}{2}\right) \cos \left(\frac{C_{1}+C_{2}}{2 C}\right) \theta$

Hence,

$$
\text { Upon rearranging, we obtained }
$$




$$
\frac{d x_{1}}{d t}=-\left(C-3 S-\frac{W_{s}}{2}\right)\left(\sin \left(\frac{2 C-6 S-W_{s}}{2 C}\right) \theta\right)\left(\frac{2 C-6 S-W_{s}}{2 C}\right) \frac{d \theta}{d t}
$$

548 Similarly, we get

$$
\frac{d y_{1}}{d t}=\left(C-3 S-\frac{W_{s}}{2}\right)\left(\cos \left(\frac{2 C-6 S-W_{s}}{2 C}\right) \theta\right)\left(\frac{2 C-6 S-W_{s}}{2 C}\right) \frac{d \theta}{d t}
$$

$$
V_{1}^{2}=\left(\frac{d x_{1}}{d t}\right)^{2}+\left(\frac{d y_{1}}{d t}\right)^{2}=\frac{1}{4}\left(2 C-6 S-W_{s}\right)^{2}\left(\frac{2 C-6 S-W_{s}}{C}\right)^{2}\left(\frac{d \theta}{d t}\right)^{2}
$$

Similarly, the expression for velocity $V_{2}$ is evaluated as

$$
V_{2}^{2}=\left(\frac{d x_{2}}{d t}\right)^{2}+\left(\frac{d y_{2}}{d t}\right)^{2}
$$

552 Where,

$$
x_{2}=x_{1}+S \cos \psi_{2}, y_{2}=y_{1}+S \sin \psi_{2}
$$

553

On substituting the expressions of $\psi_{2}$ from Eq. (A.1) in Eq. (A.12), we get

$$
\begin{aligned}
& x_{2}=\left(C-3 S-\frac{W_{s}}{2}\right) \cos \left(\frac{\theta_{1}+\theta_{2}}{2}\right)+S \cos \left(\frac{\theta_{1}+\theta_{2}+\theta_{3}+\theta_{4}}{2}\right) \\
& =\left(C-3 S-\frac{W_{s}}{2}\right) \cos \left(\frac{C_{1}+C_{2}}{2 C}\right) \theta+S \cos \left(\frac{C_{1}+C_{2}+C_{3}+C_{4}}{2 C}\right) \theta
\end{aligned}
$$

554

Hence,

$\frac{d x_{2}}{d t}=\frac{d x_{2}}{d \theta} \times \frac{d \theta}{d t}=-\left(\begin{array}{l}\left(C-3 S-\frac{W_{s}}{2}\right) \sin \left(\frac{C_{1}+C_{2}}{2 C}\right) \theta\left(\frac{C_{1}+C_{2}}{2 C}\right) \\ +S \sin \left(\frac{C_{1}+C_{2}+C_{3}+C_{4}}{2 C}\right) \theta\left(\frac{C_{1}+C_{2}+C_{3}+C_{4}}{2 C}\right)\end{array}\right) \frac{d \theta}{d t}$ 


$$
\left(\frac{d x_{2}}{d t}\right)^{2}=\left(\begin{array}{l}
\left(C-3 S-\frac{W_{s}}{2}\right)^{2} \sin ^{2}\left(\frac{C_{1}+C_{2}}{2 C}\right) \theta\left(\frac{C_{1}+C_{2}}{2 C}\right)^{2} \\
+S^{2} \sin ^{2}\left(\frac{C_{1}+C_{2}+C_{3}+C_{4}}{2 C}\right) \theta\left(\frac{C_{1}+C_{2}+C_{3}+C_{4}}{2 C}\right)^{2} \\
+2\left(C-3 S-\frac{W_{s}}{2}\right) \sin \left(\frac{C_{1}+C_{2}}{2 C}\right) \theta\left(\frac{C_{1}+C_{2}}{2 C}\right) \\
\times S \sin \left(\frac{C_{1}+C_{2}+C_{3}+C_{4}}{2 C}\right) \theta\left(\frac{C_{1}+C_{2}+C_{3}+C_{4}}{2 C}\right)
\end{array}\right)\left(\frac{d \theta}{d t}\right)^{2}
$$

$$
\left(\frac{d y_{2}}{d t}\right)^{2}=\left(\begin{array}{l}
\left(C-3 S-\frac{W_{s}}{2}\right)^{2} \cos ^{2}\left(\frac{C_{1}+C_{2}}{2 C}\right) \theta\left(\frac{C_{1}+C_{2}}{2 C}\right)^{2} \\
+S^{2} \cos ^{2}\left(\frac{C_{1}+C_{2}+C_{3}+C_{4}}{2 C}\right) \theta\left(\frac{C_{1}+C_{2}+C_{3}+C_{4}}{2 C}\right)^{2} \\
+2\left(C-3 S-\frac{W_{s}}{2}\right) \cos \left(\frac{C_{1}+C_{2}}{2 C}\right) \theta\left(\frac{C_{1}+C_{2}}{2 C}\right) \\
\times S \cos \left(\frac{C_{1}+C_{2}+C_{3}+C_{4}}{2 C}\right) \theta\left(\frac{C_{1}+C_{2}+C_{3}+C_{4}}{2 C}\right)
\end{array}\right)\left(\frac{d \theta}{d t}\right)^{2}
$$

$$
\left.\begin{array}{rl}
V_{2}^{2}=\left(\frac{d x_{2}}{d t}\right)^{2}+\left(\frac{d y_{2}}{d t}\right)^{2} \\
=\left(\begin{array}{l}
\left(C-3 S-\frac{W_{s}}{2}\right)^{2}\left(\frac{C_{1}+C_{2}}{2 C}\right)^{2}+S^{2}\left(\frac{C_{1}+C_{2}+C_{3}+C_{4}}{2 C}\right)^{2} \\
+2 S\left(C-3 S-\frac{W_{s}}{2}\right)\left(\frac{C_{1}+C_{2}}{2 C}\right)\left(\frac{C_{1}+C_{2}+C_{3}+C_{4}}{2 C}\right) \\
\times\left(\begin{array}{c}
\sin \left(\frac{C_{1}+C_{2}}{2 C}\right) \theta \sin \left(\frac{C_{1}+C_{2}+C_{3}+C_{4}}{2 C}\right) \theta \\
+\cos \left(\frac{C_{1}+C_{2}}{2 C}\right) \theta \cos \left(\frac{C_{1}+C_{2}+C_{3}+C_{4}}{2 C}\right) \theta
\end{array}\right)
\end{array}\right)\left(\frac{d \theta}{d t}\right)^{2}
\end{array}\right)
$$


By using the trigonometric identity: $\sin A \sin B+\cos A \cos B=\cos (A-B)$, we get

$$
V_{2}^{2}=\left(\begin{array}{l}
\left(C-3 S-\frac{W_{s}}{2}\right)^{2}\left(\frac{C_{1}+C_{2}}{2 C}\right)^{2} \\
+S^{2}\left(\frac{C_{1}+C_{2}+C_{3}+C_{4}}{2 C}\right)^{2} \\
+2 S\left(C-3 S-\frac{W_{s}}{2}\right)\left(\frac{C_{1}+C_{2}}{2 C}\right) \\
\times\left(\frac{C_{1}+C_{2}+C_{3}+C_{4}}{2 C}\right)\left(\cos \left(\frac{-C_{3}-C_{4}}{2 C}\right) \theta\right)
\end{array}\right)\left(\frac{d \theta}{d t}\right)^{2}
$$

On inheriting the expressions of $C_{1}, C_{2}, C_{3}$, and $C_{4}$ from Eq. (A.3) and substituting 560 in Eq. (A.18), we get

$$
V_{2}^{2}=\frac{1}{4}\left(\begin{array}{l}
\left(2 C-6 S-W_{s}\right)^{2}\left(\frac{2 C-6 S-W_{s}}{C}\right)^{2} \\
+S^{2}\left(\frac{4 C-10 S-2 W_{s}}{C}\right)^{2} \\
+2 S\left(2 C-6 S-W_{s}\right)\left(\frac{2 C-6 S-W_{s}}{C}\right) \\
\times\left(\frac{4 C-10 S-2 W_{s}}{2 C}\right)\left(\cos \left(\frac{4 S+W_{s}-2 C}{2 C}\right) \theta\right)
\end{array}\right)\left(\frac{d \theta}{d t}\right)^{2}
$$

Similarly, by using the following expressions for $V_{3}$, and $V_{4}$

$$
V_{3}^{2}=\left(\frac{d x_{3}}{d t}\right)^{2}+\left(\frac{d y_{3}}{d t}\right)^{2}, V_{4}^{2}=\left(\frac{d x_{4}}{d t}\right)^{2}+\left(\frac{d y_{4}}{d t}\right)^{2}
$$

562 Where,

$x_{3}=x_{2}+S \cos \psi_{3}, y_{3}=y_{2}+S \sin \psi_{3}$

$x_{4}=x_{3}+S \cos \psi_{4}, y_{4}=y_{3}+S \sin \psi_{4}$

We get, 


$$
V_{3}^{2}=\frac{1}{4}\left(\begin{array}{l}
\left(2 C-6 S-W_{s}\right)^{2}\left(\frac{2 C-6 S-W_{s}}{2 C}\right)^{2} \\
+S^{2}\left(\frac{10 C-22 S-5 W_{s}}{C}\right)^{2} \\
+2 S\left(2 C-6 S-W_{s}\right)\left(\frac{2 C-6 S-W_{s}}{C}\right) \\
\times\left(\frac{10 C-22 S-5 W_{s}}{2 C}\right)\left(\cos \left(\frac{16 S+4 W_{s}-8 C}{2 C}\right) \theta\right)
\end{array}\right)\left(\frac{d \theta}{d t}\right)^{2}
$$

565

and,

$$
V_{4}^{2}=\frac{1}{4}\left(\begin{array}{l}
(2 C-6 S-W)^{2}\left(\frac{2 C-6 S-W_{s}}{2 C}\right)^{2} \\
+S^{2}\left(\frac{18 C-34 S-9 W_{s}}{C}\right)^{2} \\
+2 S(2 C-6 S-W)\left(\frac{2 C-6 S-W_{s}}{C}\right) \\
\times\left(\frac{18 C-34 S-9 W_{s}}{2 C}\right)\left(\cos \left(\frac{28 S+8 W_{s}-16 C}{2 C}\right) \theta\right)
\end{array}\right)\left(\frac{d \theta}{d t}\right)^{2}
$$

The deduced expressions of velocities $V_{1}, V_{2}, V_{3}$, and $V_{4}$ are substituted in Eq.

\section{Appendix B: Expressions of terms $\left(Z_{1}-Z_{4}\right)$}

The required expressions of the terms $\left(Z_{1}-Z_{4}\right)$ appeared in the nonlinear dynamic governing equation (Eq. 23) are as follows: 


$$
\begin{aligned}
& {\left[\left(2 C-6 S-W_{S}\right)^{2}\left(\frac{2 C-6 S-W_{s}}{C}\right)^{2}\right]} \\
& \left(2 C-6 S-W_{s}\right)^{2}\left(\frac{2 C-6 S-W_{s}}{C}\right)^{2}+S^{2}\left(\frac{4 C-10 S-2 W_{s}}{C}\right)^{2} \\
& +\left[+2 S\left(2 C-6 S-W_{s}\right)\left(\frac{2 C-6 S-W_{s}}{C}\right)\left(\frac{4 C-10 S-2 W_{s}}{2 C}\right) \cos \left(\frac{4 S+W_{s}-2 C}{2 C}\right) \theta\right. \\
& Z_{1}=\left[\begin{array}{c}
\left(2 C-6 S-W_{s}\right)^{2}\left(\frac{2 C-6 S-W_{s}}{C}\right)^{2}+S^{2}\left(\frac{10 C-22 S-5 W_{s}}{C}\right)^{2} \\
+2 S\left(2 C-6 S-W_{s}\right)\left(\frac{2 C-6 S-W_{s}}{C}\right)\left(\frac{10 C-22 S-5 W_{s}}{2 C}\right) \cos \left(\frac{16 S+4 W_{s}-8 C}{2 C}\right) \theta
\end{array}\right] \\
& {\left[\left(2 C-6 S-W_{s}\right)^{2}\left(\frac{2 C-6 S-W_{s}}{C}\right)^{2}+S^{2}\left(\frac{18 C-34 S-2 W_{s}}{C}\right)^{2}\right.} \\
& +\left[+2 S\left(2 C-6 S-W_{s}\right)\left(\frac{2 C-6 S-W_{s}}{C}\right)\left(\frac{18 C-34 S-2 W_{s}}{2 C}\right) \cos \left(\frac{28 S+8 W_{s}-16 C}{2 C}\right) \theta\right] \\
& {\left[\begin{array}{l}
2 S\left(2 C-6 S-W_{s}\right)\left(\frac{2 C-6 S-W_{s}}{C}\right)\left(\frac{4 C-10 S-2 W_{s}}{2 C}\right) \\
\sin \left(\frac{4 S+W_{s}-2 C}{2 C}\right) \theta\left(\frac{4 S+W_{s}-2 C}{2 C}\right)
\end{array}\right]} \\
& Z_{2}=\left[\begin{array}{l}
2 S\left(2 C-6 S-W_{s}\right)\left(\frac{2 C-6 S-W_{s}}{C}\right)\left(\frac{10 C-22 S-5 W_{s}}{2 C}\right) \\
\sin \left(\frac{16 S+4 W_{s}-8 C}{2 C}\right) \theta\left(\frac{16 S+4 W_{s}-8 C}{2 C}\right)
\end{array}\right] \\
& {\left[-\left[\begin{array}{l}
2 S\left(2 C-6 S-W_{s}\right)\left(\frac{2 C-6 S-W_{s}}{C}\right)\left(\frac{18 C-34 S-2 W_{s}}{2 C}\right) \\
\sin \left(\frac{28 S+8 W_{s}-16 C}{2 C}\right) \theta\left(\frac{28 S+8 W_{s}-16 C}{2 C}\right)
\end{array}\right]\right]}
\end{aligned}
$$$$
573
$$ 


$$
\begin{aligned}
Z_{3}= & {\left[\begin{array}{l}
K_{b f}+K_{b s}\left(\frac{W_{s}}{C^{2}}\right)\left(2 C-W_{s}\right)+K_{b s}\left(\frac{W_{s}}{C^{2}}\right)\left(2 C-W_{s}-2 S\right) \\
\left.+K_{b s}\left(\frac{W_{s}}{C^{2}}\right)\left(2 C-W_{s}-3 S\right)+K_{b s}\left(\frac{W_{s}}{C^{2}}\right)\left(2 C-W_{s}-4 S\right)\right]
\end{array}\right.} \\
Z_{4}= & {\left[\begin{array}{l}
2 \lambda_{p}^{2} \sin \left(\frac{\theta}{2}\right)\left[\frac{\cos \left(\frac{\theta}{2}\right) \theta-2 \sin \left(\frac{\theta}{2}\right)}{\theta^{3}}\right]+\frac{1}{8 \lambda^{4}}\left[\frac{2 \sin \left(\frac{\theta}{2}\right) \theta-\theta^{2} \cos \left(\frac{\theta}{2}\right)}{\sin ^{3}\left(\frac{\theta}{2}\right)}\right] \\
-2 \lambda_{p}^{4} \sin \left(\frac{\theta}{2}\right)\left[\frac{\cos \left(\frac{\theta}{2}\right) \theta-2 \sin \left(\frac{\theta}{2}\right)}{\theta^{3}}\right] E^{2} \frac{\varepsilon}{\mu}
\end{array}\right] }
\end{aligned}
$$

\section{References}

OA Araromi, Irina Gavrilovich, Jun Shintake, Samuel Rosset, and HR Shea. Towards a deployable satellite gripper based on multisegment dielectric elastomer minimum energy structures. In Electroactive Polymer Actuators and Devices (EAPAD) 2014, volume 9056, page 90562G. International Society for Optics and Photonics, 2014. Zhe Chen, Xueya Liang, Tonghao Wu, Tenghao Yin, Yuhai Xiang, and Shaoxing Qu. Pneumatically actuated soft robotic arm for adaptable grasping. Acta Mechanica Solida Sinica, 31(5):608-622, 2018.

Stefania Colonnelli, Giuseppe Saccomandi, and Giuseppe Zurlo. The role of material behavior in the performances of electroactive polymer energy harvesters. Journal of Polymer Science Part B: Polymer Physics, 53(18):1303-1314, 2015.

Hu-liang Dai and Lin Wang. Nonlinear oscillations of a dielectric elastomer membrane subjected to in-plane stretching. Nonlinear Dynamics, 82(4):1709-1719, 2015.

Huliang Dai, Jiangjiang Zou, and Lin Wang. Effect of initial stretch ratio on the electromechanical responses of dielectric elastomer actuators. Applied Physics A, 122(5):1-6, 2016.

M Follador, AT Conn, and J Rossiter. Bistable minimum energy structures (bimes) for binary robotics. Smart Materials and Structures, 24(6):065037, 2015.

Guo-Ying Gu, Jian Zhu, Li-Min Zhu, and Xiangyang Zhu. A survey on dielectric elastomer actuators for soft robots. Bioinspiration \& biomimetics, 12(1):011003, 2017.

M M Joglekar. An energy-based approach to extract the dynamic instability parameters of dielectric elastomer actuators. Journal of Applied Mechanics, 81(9):091010, 2014.

Aman Khurana, Ajay Kumar, Santosh Kumar Raut, Atul Kumar Sharma, and MM Joglekar. Effect of viscoelasticity on the nonlinear dynamic behavior of di- 
electric elastomer minimum energy structures. International Journal of Solids and Structures, 2020.

Aman Khurana, Ajay Kumar, Atul Kumar Sharma, and MM Joglekar. Effect of polymer chains entanglements, crosslinks and finite extensibility on the nonlinear dynamic oscillations of dielectric viscoelastomer actuators. Nonlinear Dynamics, pages $1-25,2021 \mathrm{a}$

Aman Khurana, Atul Kumar Sharma, and MM Joglekar. Nonlinear oscillations of electrically driven aniso-visco-hyperelastic dielectric elastomer minimum energy structures. Nonlinear Dynamics, pages 1-23, 2021 b.

Guggi Kofod, Mika Paajanen, and Siegfried Bauer. Self-organized minimum-energy structures for dielectric elastomer actuators. Applied Physics A, 85(2):141-143, 2006.

Guggi Kofod, Werner Wirges, Mika Paajanen, and Siegfried Bauer. Energy minimization for self-organized structure formation and actuation. Applied Physics Letters, 90(8):081916, 2007.

Matthias Kollosche, Guggi Kofod, Zhigang Suo, and Jian Zhu. Temporal evolution and instability in a viscoelastic dielectric elastomer. Journal of the Mechanics and Physics of Solids, 76:47-64, 2015.

William Lai, Ashraf F Bastawros, and Wei Hong. Out-of-plane motion of a planar dielectric elastomer actuator with distributed stiffeners. In Electroactive Polymer Actuators and Devices (EAPAD) 2012, volume 8340, page 834011. International Society for Optics and Photonics, 2012.

Hao Liu, Xiaolan Wang, Toshiyuki Nakata, and Kazuyuki Yoshida. Aerodynamics and flight stability of bio-inspired, flapping-wing micro air vehicles. In $\mathrm{Au}$ tonomous Control Systems and Vehicles, pages 145-157. Springer, 2013.

Lei Liu, Hualing Chen, Bo Li, Yanjie Wang, and Dichen Li. Thermal and strainstiffening effects on the electromechanical breakdown strength of dielectric elastomers. Applied Physics Letters, 107(6):062906, 2015.

Kai Luo, Qiang Tian, and Haiyan Hu. Dynamic modeling, simulation and design of smart membrane systems driven by soft actuators of multilayer dielectric elastomers. Nonlinear Dynamics, 102(3):1463-1483, 2020.

Kevin Y Ma, Pakpong Chirarattananon, Sawyer B Fuller, and Robert J Wood. Controlled flight of a biologically inspired, insect-scale robot. Science, 340(6132): 603-607, 2013.

Benjamin O'Brien, Thomas McKay, Emilio Calius, Shane Xie, and Iain Anderson. Finite element modelling of dielectric elastomer minimum energy structures. Applied Physics A, 94(3):507-514, 2009.

Harold S Park, Zhigang Suo, Jinxiong Zhou, and Patrick A Klein. A dynamic finite element method for inhomogeneous deformation and electromechanical instability of dielectric elastomer transducers. International Journal of Solids and Structures, 49(15):2187-2194, 2012.

Ron Pelrine, Roy Kornbluh, Qibing Pei, and Jose Joseph. High-speed electrically actuated elastomers with strain greater than 100\%. Science, 287(5454):836-839, 2000.

Samuel Rosset, Oluwaseun A Araromi, Jun Shintake, and Herbert R Shea. Model and design of dielectric elastomer minimum energy structures. Smart Materials 
and Structures, 23(8):085021, 2014.

S Sasikala, KT Madhavan, G Ramesh, S Saggar, P Predeep, and P Chowdhury. Electro-mechanical response to the harmonic actuation of the pneumatically coupled dielectric elastomer based actuators with and without load. International Journal of Solids and Structures, 110:58-66, 2017.

Atul Kumar Sharma, S Bajpayee, DM Joglekar, and MM Joglekar. Dynamic instability of dielectric elastomer actuators subjected to unequal biaxial prestress. Smart Materials and Structures, 26(11):115019, 2017.

Junjie Sheng, Hualing Chen, Bo Li, and Yongquan Wang. Nonlinear dynamic characteristics of a dielectric elastomer membrane undergoing in-plane deformation. Smart Materials and Structures, 23(4):045010, 2014.

Renata M Soares and Paulo B Gonçalves. Nonlinear vibrations of a rectangular hyperelastic membrane resting on a nonlinear elastic foundation. Meccanica, 53(4): 937-955, 2018.

Renata M Soares, Pedro FT Amaral, Frederico MA Silva, and Paulo Batista Gonçalves. Nonlinear breathing motions and instabilities of a pressure-loaded spherical hyperelastic membrane. Nonlinear Dynamics, 99(1):351-372, 2020.

Steven H Strogatz. Nonlinear dynamics and chaos: with applications to physics, biology, chemistry, and engineering. CRC Press, 2018.

Dafeng Tang, CW Lim, Ling Hong, Jun Jiang, and Siu Kai Lai. Analytical asymptotic approximations for large amplitude nonlinear free vibration of a dielectric elastomer balloon. Nonlinear Dynamics, 88(3):2255-2264, 2017.

$\mathrm{H}$ Vatanjou, Y Hojjat, and M Karafi. Nonlinear dynamic analysis of dielectric elastomer minimum energy structures. Applied Physics A, 125(9):583, 2019.

Jin Wang, Thao D Nguyen, and Harold S Park. Electrostatically driven creep in viscoelastic dielectric elastomers. Journal of Applied Mechanics, 81(5):051006, 2014.

Michael Wissler and Edoardo Mazza. Modeling and simulation of dielectric elastomer actuators. Smart Materials and structures, 14(6):1396, 2005.

Junshi Zhang and Hualing Chen. Voltage-induced beating vibration of a dielectric elastomer membrane. Nonlinear Dynamics, 100:2225-2239, 2020.

Junshi Zhang, Hualing Chen, and Dichen Li. Pinnacle elimination and stability analyses in nonlinear oscillation of soft dielectric elastomer slide actuators. Nonlinear Dynamics, 94(3):1907-1920, 2018.

Junshi Zhang, Liling Tang, Lei Liu, Jianwen Zhao, Zhichun Yang, and Pengfei Li. Modeling of humidity effect on electromechanical properties of viscoelastic dielectric elastomer. International Journal of Mechanical Sciences, 193:106177, 2021.

Jianwen Zhao, Junyang Niu, David McCoul, Jinsong Leng, and Qibing Pei. A rotary joint for a flapping wing actuated by dielectric elastomers: design and experiment. Meccanica, 50(11):2815-2824, 2015a.

Jianwen Zhao, Junyang Niu, David McCoul, Zhi Ren, and Qibing Pei. Phenomena of nonlinear oscillation and special resonance of a dielectric elastomer minimum energy structure rotary joint. Applied Physics Letters, 106(13):133504, 2015b.

Jianwen Zhao, Shu Wang, David McCoul, Zhiguang Xing, Bo Huang, Liwu Liu, and Jinsong Leng. Bistable dielectric elastomer minimum energy structures. Smart 
Materials and structures, 25(7):075016, 2016a.

Jianwen Zhao, Shu Wang, Zhiguang Xing, David McCoul, Junyang Niu, Bo Huang, Liwu Liu, and Jinsong Leng. Equivalent dynamic model of demes rotary joint. Smart Materials and structures, 25(7):075025, $2016 \mathrm{~b}$.

Zhentao Zhao, Datian Niu, Hongwu Zhang, and Xuegang Yuan. Nonlinear dynamics of loaded visco-hyperelastic spherical shells. Nonlinear Dynamics, 101(2):911933, 2020.

Lei Zhou, Shibin Wang, Linan Li, and Yibin Fu. An evaluation of the gent and gentgent material models using inflation of a plane membrane. International Journal of Mechanical Sciences, 146:39-48, 2018. 